\title{
DIMERS ON RAIL YARD GRAPHS
}

\author{
CÉDRIC BOUTILLIER, JÉRÉMIE BOUTTIER, GUILLAUME CHAPUY, \\ SYLVIE CORTEEL, AND SANJAY RAMASSAMY
}

\begin{abstract}
We introduce a general model of dimer coverings of certain plane bipartite graphs, which we call rail yard graphs (RYG). The transfer matrices used to compute the partition function are shown to be isomorphic to certain operators arising in the so-called boson-fermion correspondence. This allows to reformulate the RYG dimer model as a Schur process, i.e. as a random sequence of integer partitions subject to some interlacing conditions.

Beyond the computation of the partition function, we provide an explicit expression for all correlation functions or, equivalently, for the inverse Kasteleyn matrix of the RYG dimer model. This expression, which is amenable to asymptotic analysis, follows from an exact combinatorial description of the operators localizing dimers in the transfer-matrix formalism, and then a suitable application of Wick's theorem.

Plane partitions, domino tilings of the Aztec diamond, pyramid partitions, and steep tilings arise as particular cases of the RYG dimer model. For the Aztec diamond, we provide new derivations of the edge-probability generating function, of the biased creation rate, of the inverse Kasteleyn matrix and of the arctic circle theorem.
\end{abstract}

\section{INTRODUCTION}

The two-dimensional dimer model is arguably the most studied exactly solvable model in statistical mechanics (note that it encompasses, in a sense, the equally well-known two-dimensional Ising model), see for instance [Kas67, Chapter 5] for a review of the seminal works of Kasteleyn, Temperley and Fisher, and the introduction of All14 for a nice survey of the more recent literature. Dimer configurations are also known as perfect matchings in combinatorics and theoretical computer science. Actually, perhaps the oldest exact solution of a 2D dimer model is MacMahon's enumeration of plane partitions [Mac04, as these were later identified with lozenge tilings, or alternatively dimer configurations on the hexagonal lattice.

Kasteleyn's method allows to reduce the problem of computing the partition function (and the correlation functions) of the dimer model on any finite weighted planar graph (assuming that the dimers interact only through their hard-core repulsion) to the evaluation of a determinant (or Pfaffian) whose size is linear in the number of vertices of the graph. Under the usual assumption that the graph is periodic in two directions, one can then evaluate this determinant and take the thermodynamic limit to obtain the free energy, study phase transitions, etc. In this paper, we consider a dimer model on a new family of graphs, called rail yard graphs, which are periodic in one direction but not in the other.

Date: June 30, 2015.

We acknowledge financial support from the Ville de Paris via Projet Émergences "Combinatoire à Paris" (JB, GC, SC), from the Monahan Foundation via a Monahan Science Fellowship (SR) and from the Agence Nationale de la Recherche via the grants ANR-08-JCJC-0011 "IComb" (SC), ANR-10-BLAN-0123 "MAC2" (CB), ANR-12-JS02-0001 "Cartaplus" (JB, GC), ANR-14-CE250014 "GRAAL" (JB). JB acknowledges the hospitality of LIAFA. SR acknowledges the hospitality and financial support of the Erwin Schödinger Institute in Vienna, where part of this work was done. 
One of our motivations is that the rail yard graph dimer model encompasses both the plane partitions mentioned above and another celebrated model, namely domino tilings of the Aztec diamond [EKLP92a, EKLP92b] (corresponding to, roughly speaking, dimer configurations on the portion of the square lattice fitting into a large square tilted by $45^{\circ}$ ). What relates these two models is that they can be seen as Schur processes OR03, that is to say random sequences of integer partitions whose transition probabilities are given by Schur functions. If the relation between plane partitions and Schur processes was explicited by Okounkov and Reshetikhin, the case of the Aztec diamond appears implicitly in Joh02 and has, to the best of our knowledge, remained in such implicit form until BCC14, of which this paper is a continuation (see below). The interest of making the connection between dimer models and Schur processes explicit is that it allows to use an operator formalism coming from the boson-fermion correspondence (see the references given at the beginning of Section 3 which is both powerful and intuitive, as the operators are nothing but transfer matrices or observables satisfying some particularly simple commutation relations. Furthermore it allows us to say that the RYG dimer model forms another situation, besides the 2D Ising model Dub11, where "bosonization" works at an exact discrete level. The rail yard graph dimer model corresponds essentially to the most general Schur process with nonnegative transition probabilities.

Before describing our work in more detail, let us further discuss some history and background behind it. Bender and Knuth BK72] made the link between plane partitions and the Robinson-Schensted-Knuth correspondence, see also Sta99, Chapter 7]. Okounkov Oko01, Oko02] used the boson-fermion correspondence to define the so-called Schur measure over integer partitions, and study its correlation functions. The Schur process [OR03, OR07] is a time-dependent version of this measure, that can also be viewed as a system of particles with certain dynamics. It contains as a special case a generalization of plane partitions, namely plane partitions with an evolving "back wall". Numerous papers followed on this subject Bor07, BF14, Bor11, BMRT12, $\mathrm{BBB}^{+} 14, \mathrm{BF} 15$ ] and on its extension to the Hall-Littlewood and Macdonald cases [Vul07, FW09, Vul09, Oka10, CSV11, BC14.

In [BCC14, three authors of the present paper introduced a general class of domino tilings called steep tilings, encompassing both tilings of the Aztec diamond and the so-called pyramid partitions Ken05, You09. It was shown in BCC14 that steep tilings also correspond to Schur processes and, using the vertex operator formalism, their partition functions (of the "hook formula" type) were computed for a variety of boundary conditions. Since both (generalized) plane partitions and steep tilings are special instances of the Schur process, it is then natural to ask if there is a more general model of tilings or dimer coverings that would reformulate the Schur process in full generality, at least when the number of underlying parameters is finite. Such a model was sketched in [BCC14, Section 7], that can be viewed as a preliminary attempt at what we reach in the present paper.

The rail yard graphs (RYG) that we introduce here are infinite bipartite plane graphs, obtained by the "concatenation" of column-shaped elementary graphs, and come with a family of admissible dimer coverings. The RYG dimer model is then a probability measure over such coverings. The elementary graphs can be of four types that correspond to the four possible types of "atomic" transitions in the Schur process. For the special families of RYG that correspond to the special families of Schur processes considered in OR03, BCC14, we recover generalized plane partitions and steep tilings, respectively. As we hope will be apparent in this paper, RYG provide a nice and natural formulation of the Schur process in terms of dimers, in a well-adapted system of coordinates, much simpler than the 
one from [BCC14, Section 7]. Having shown the correspondence between rail yard graphs and Schur process, we can then apply the same classical tools as in OR03. to get explicitly the partition functions in a nice (hook-type) product form. Even more, we can interpret these partition functions in terms of a combinatorial parameter related to the flip operation on coverings.

Beyond the partition function, we compute all the dimer correlation functions, which requires the introduction of suitably defined observables (or constrained transfer matrices) that enable us to localize, in the algebraic setting, a given set of dimers. To prevent any confusion, let us note that the particle correlations computed in OR03 for the Schur process, when translated in terms of RYG, give only a special case of this result. Indeed, as we will see, there are three kinds of dimers in a RYG, and particles correspond to one of the three kinds (so in our setting the correlations results of OR03 only describe correlations between dimers of the first kind). Once the observables are constructed, we use classical fermionic tools such as Wick's formula to evaluate the correlation functions in an explicit determinantal form. We also make the connection with the general Kasteleyn theory: it is a general fact that correlations between dimers on plane bipartite graphs have a determinantal form, underlaid by an inverse of the so-called Kasteleyn matrix of the model. For RYG, we show that the determinantal form we obtain by our approach indeed gives an inverse Kasteleyn matrix, as was remarked in [OR07] for the case of skew plane partitions, see also [BF14, Section 5]. Our approach generalizes both this case and that of the Aztec diamond (for the so-called $q^{\text {vol }}$ weighting), treated previously in CY14 by a very tricky and somehow mysterious calculation. As further applications concerning the Aztec diamond, we rederive the so-called edge-probability generating function and biased creation rate, and the arctic circle theorem using the general saddle-point techniques of OR03.

We now present the structure of the paper. Section 2 is devoted to the basic definitions (rail yard graphs in Subsection 2.1. their dimer coverings in Subsection 2.2. flips in Subsection 2.3 and to the statement of our main results, namely the expression for the partition function (Subsection 2.4) and for the dimer correlation functions (Subsection 2.5). Section 3 introduces bosonic operators (Subsection 3.1) that act as transfer matrices in the RYG dimer model (Subsection 3.2), allowing to compute efficiently the partition function (Subsection 3.3). Section 4 considers fermionic operators (Subsection 4.1) that play the role of observables in the RYG dimer model (Subsection 4.2). Rewriting the correlation functions in the "Heisenberg picture" (Subsection 4.3), we derive their expression in the form of a determinant (Subsection 4.4), before making the connection with Kasteleyn's theory (Subsection 4.5). Section 5 discusses the previously known cases: plane partitions and lozenge tilings (Subsection 5.1) and steep domino tilings (Subsection 5.2. In Section 6 we address the specific case of the Aztec diamond, for which we provide new derivations of the edge-probability generating function and biased creation rate (Subsection 6.1), of the inverse Kasteleyn matrix (Subsection 6.2) and of the arctic circle theorem (Subsection 6.3). Concluding remarks are gathered in Section 7. Some auxiliary material is given in the appendix: a combinatorial proof of the bosonic-fermionic commutation relations (Appendix A) and a rederivation of Wick's formula (Appendix B).

After the completion of this work, Alexei Borodin and Senya Shlosman informed us (by private communication) that graphs similar to those described in Section 2.1 were suggested to them by Richard Kenyon, providing a dimer interpretation of the $\alpha \beta$-paths considered in $\mathrm{BS} 10$. 


\section{BASIC DEFINITIONS AND MAIN RESULTS}

2.1. Rail yard graphs. We start by defining the underlying graph of our dimer model. We fix two integers $\ell, r$ such that $\ell \leq r$, and denote by $[\ell . . r]$ the set of integers between $\ell$ and $r$. We then consider two binary sequences indexed by the elements of $[\ell . . r]$ :

- the LR sequence $\underline{a}=\left(a_{\ell}, a_{\ell+1}, \ldots, a_{r}\right) \in\{L, R\}^{[\ell . r r]}$,

- the sign sequence $\underline{b}=\left(b_{\ell}, b_{\ell+1}, \ldots, b_{r}\right) \in\{+,-\}^{[\ell . . r]}$.

The rail yard graph associated with the integers $\ell$ and $r$, the LR sequence $a$ and the sign sequence $\underline{b}$, and denoted by $\operatorname{RYG}(\ell, r, \underline{a}, \underline{b})$, is the bipartite plane graph defined as follows. Its vertex set is $[2 \ell-1 . .2 r+1] \times(\mathbb{Z}+1 / 2)$, and we say that a vertex is even (resp. odd) if its abscissa is an even (resp. odd) integer. Each even vertex $(2 m, y), m \in[\ell . . r]$, is then incident to three edges: two horizontal edges connecting it to the odd vertices $(2 m-1, y)$ and $(2 m+1, y)$, and one diagonal edge connecting it to

- the odd vertex $(2 m-1, y+1)$ if $a_{m}=L$ and $b_{m}=+$,

- the odd vertex $(2 m-1, y-1)$ if $a_{m}=L$ and $b_{m}=-$,

- the odd vertex $(2 m+1, y+1)$ if $a_{m}=R$ and $b_{m}=+$,

- the odd vertex $(2 m+1, y-1)$ if $a_{m}=R$ and $b_{m}=-$.

Hopefully, this explains our motivations for using the symbols $L, R$ and,+- . Drawing the edges straight, the graph is indeed bipartite and plane by construction. For $e$ an edge, we write $e=(\alpha, \beta)$ to mean that $\alpha$ is the even endpoint of $e$, and $\beta$ its odd endpoint. For $v$ a vertex, we will denote by $v^{\mathrm{x}} \in \mathbb{Z}$ its abscissa, and by $v^{\mathrm{y}} \in \mathbb{Z}+1 / 2$ its ordinate.

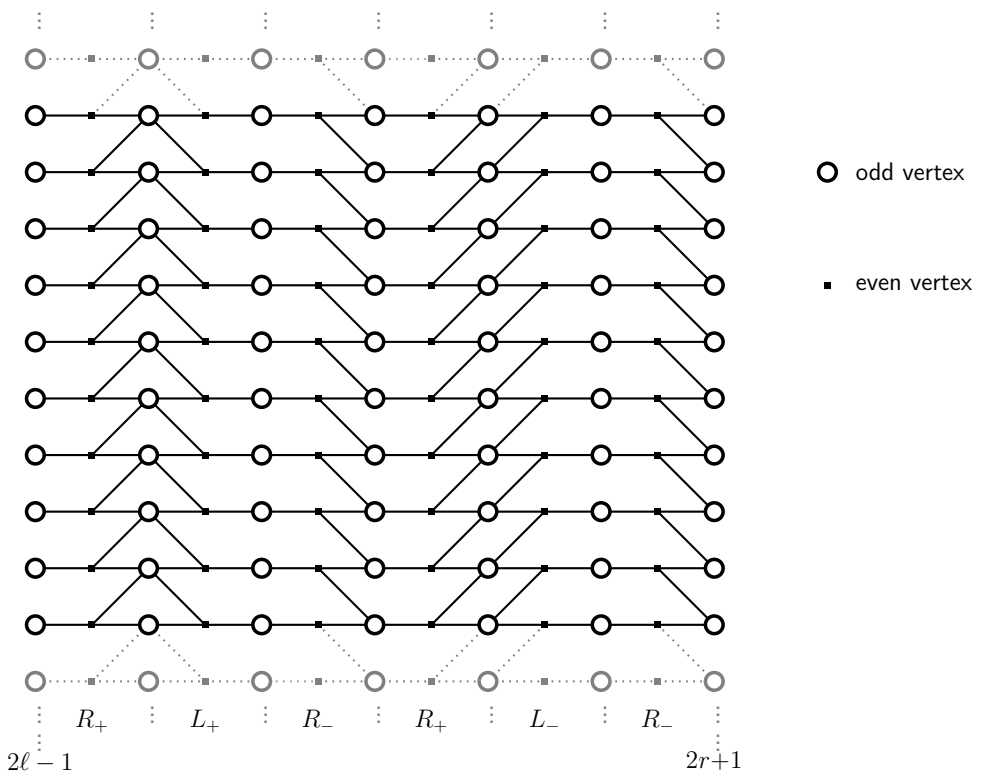

FiguRE 1. The rail yard graph associated with the LR sequence $R L R R L R$ and the sign sequence $++-+--($ with $\ell=1, r=6)$. It is infinite and periodic in the vertical direction, but finite in the horizontal direction.

Figure 1 displays the rail yard graph associated with the LR sequence $R L R R L L$ and the sign sequence $++-+--($ with $\ell=1, r=6)$. Observe that a rail yard graph is infinite and 1-periodic in the vertical direction. When $\ell=r$, the LR 
and sign sequences both consist of a single element, and the corresponding rail yard graph, which is said elementary, is of one of four possible types, see Figure 2. Given two rail yard graphs $\operatorname{RYG}(\ell, r, \underline{a}, \underline{b})$ and $\operatorname{RYG}\left(\ell^{\prime}, r^{\prime}, \underline{a}^{\prime}, \underline{b}^{\prime}\right)$ such that $\ell^{\prime}=r+1$, we define their concatenation by taking the union of their vertex and edge sets. It is nothing but the rail yard graph $\operatorname{RYG}\left(\ell, r^{\prime}, \underline{a a^{\prime}}, \underline{b b^{\prime}}\right)$ where $\underline{a}^{\prime}$ and $\underline{b b^{\prime}}$ denote the concatenations of the LR and sign sequences. Clearly, a general rail yard graph is obtained by concatenating elementary ones.
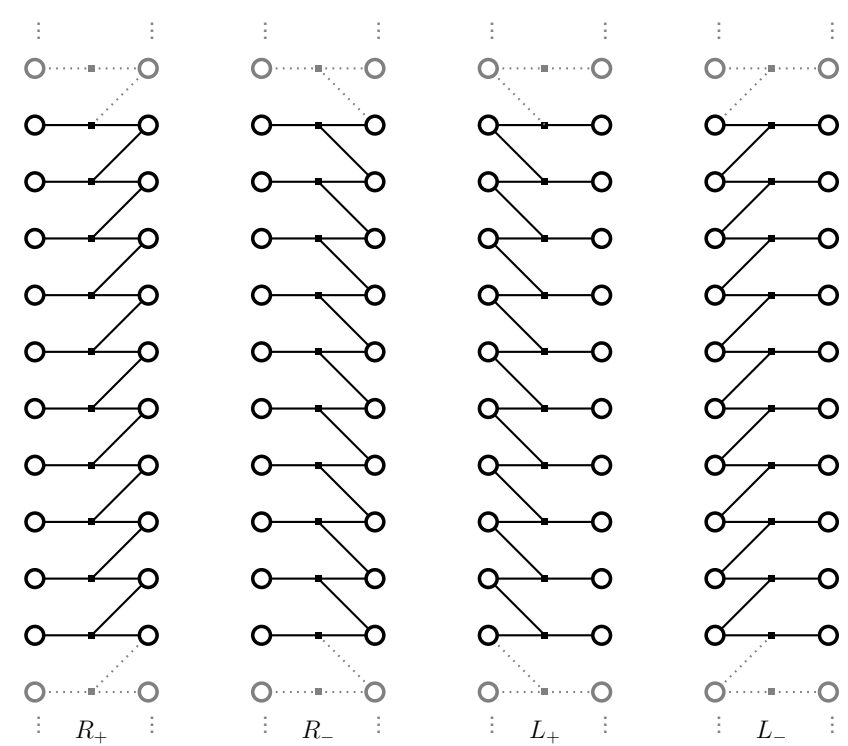

FiguRE 2. The four elementary rail yard graphs $L+, L-, R+$, and $R-$ (up to horizontal translation)

The left boundary (resp. right boundary) of a rail yard graph consists of all odd vertices with abscissa $2 \ell-1$ (resp. $2 r+1$ ). Vertices which do not belong to the boundaries are said inner. When drawn in the plane, the graph delimits some faces, and the bounded ones are called inner faces. Note that inner faces may be incident to 4,6 or 8 edges. Finally, observe that our definition works equally well if we take $\ell=-\infty$ and/or $r=+\infty$, thus considering infinite LR and sign sequences. In that case, the rail yard graph "fills" either the whole plane or a half-plane, boundaries being sent to infinity.

2.2. Admissible and pure dimer coverings. We now turn to the characterization of the configurations of our dimer model. Given a rail yard graph $\mathrm{RYG}(\ell, r, \underline{a}, \underline{b})$ with $\ell, r$ finite, an admissible dimer covering is a partial matching of this graph such that:

- each inner vertex is covered (i.e. matched),

- there exists an integer $N \geq 0$ such that: any left boundary vertex $(2 \ell-1, y)$ is covered for $y>N$ and uncovered for $y<-N$, any right boundary vertex $(2 r+1, y)$ is covered for $y<-N$ and uncovered for $y>N$,

- only a finite number of diagonal edges are covered.

A pure dimer covering is an admissible dimer covering for which the second property above holds for $N=0$ : in other words the uncovered vertices are precisely the left boundary vertices with negative ordinate and the right boundary vertices with positive ordinate (see Figure 4). The fundamental dimer covering is the pure dimer covering where no diagonal edge is covered (it is not difficult to check its existence 
and uniqueness e.g. by induction on $r-\ell$ ). Observe that any admissible dimer covering coincides with the fundamental dimer covering outside a finite region. An elementary dimer covering is an admissible dimer covering of an elementary rail yard graph (see Figure 3).

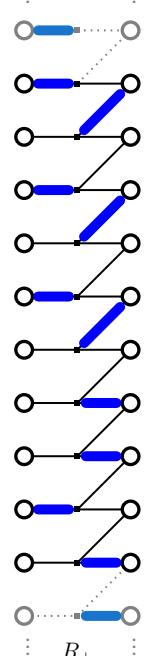

$R_{+}$
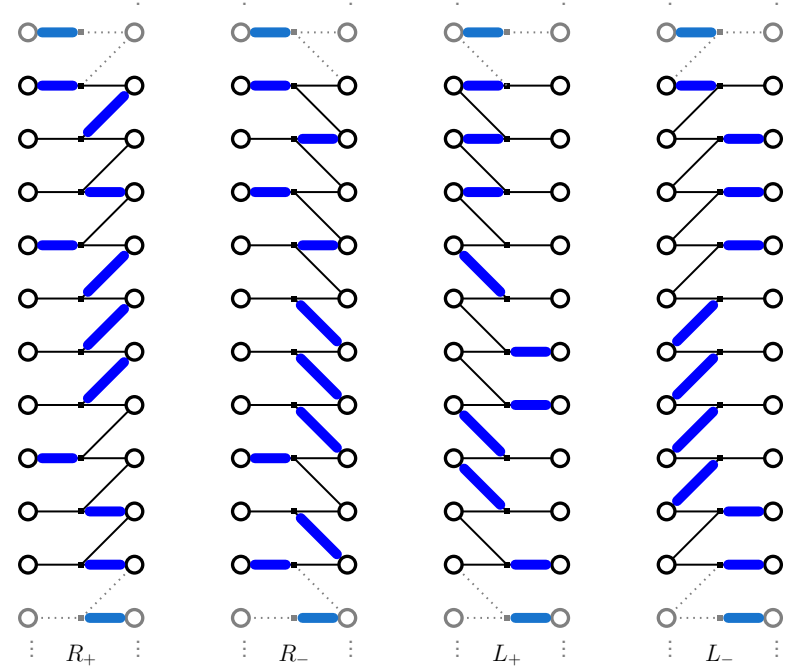

Figure 3. Some elementary dimer coverings. The underlying elementary rail yard graph has type $R+$ in the first two cases, and $R-, L+$, and $L-$, in the three others, from left to right. Outside of the displayed region, the configuration is identical towards the top (resp. bottom) on each horizontal level to what it is on the topmost (resp. bottommost) displayed level.

Similarly to rail yard graphs, admissible dimer coverings behave nicely with respect to concatenation. More precisely, consider two rail yard graphs $G=$ $\operatorname{RYG}(\ell, r, \underline{a}, \underline{b})$ and $G^{\prime}=\operatorname{RYG}\left(\ell^{\prime}, r^{\prime}, \underline{a}^{\prime}, \underline{b}^{\prime}\right)$ which are concatenable (i.e. $\ell^{\prime}=r+1$ ) and let $G G^{\prime}$ be their concatenation. Let $C$ and $C^{\prime}$ be admissible dimer coverings of respectively $G$ and $G^{\prime}$ : we say that $C$ and $C^{\prime}$ are compatible if, for each $y \in \mathbb{Z}+1 / 2$, the vertex $(2 r+1, y)=\left(2 \ell^{\prime}-1, y\right)$ is covered in $C$ if and only if it is not covered in $C^{\prime}$. In that case, by taking the union of $C$ and $C^{\prime}$, we obtain an admissible dimer covering of $G G^{\prime}$, which we denote by $C C^{\prime}$. Conversely, any admissible dimer covering can be decomposed as the concatenation of elementary dimer coverings which are sequentially compatible.

It is also interesting to consider the limiting cases $\ell=-\infty$ and/or $r=+\infty$, which requires a slight adaptation of our definitions. An admissible (resp. a pure, resp. the fundamental) dimer covering is then a matching such that each inner vertex is covered, and such that there exists finite integers $\ell^{\prime}, r^{\prime}$ such that :

- inside the strip $\left[2 \ell^{\prime}-1,2 r^{\prime}+1\right] \times \mathbb{R}$, we see an admissible (resp. a pure, resp. the fundamental) dimer covering in the previous sense,

- outside this strip, all covered edges are horizontal.

(Note that this definition works in all situations: it coincides with the previous one when $\ell, r$ are both finite.)

Our motivation for considering pure dimer coverings of rail yard graphs is that we recover several well-known dimer models as specializations. For instance, taking $r-\ell=2 n$, a LR sequence of the form $L R L R L R \cdots$ and a sign sequence of the form $+-+-+-\cdots$, the corresponding pure dimer configurations are in bijection with 


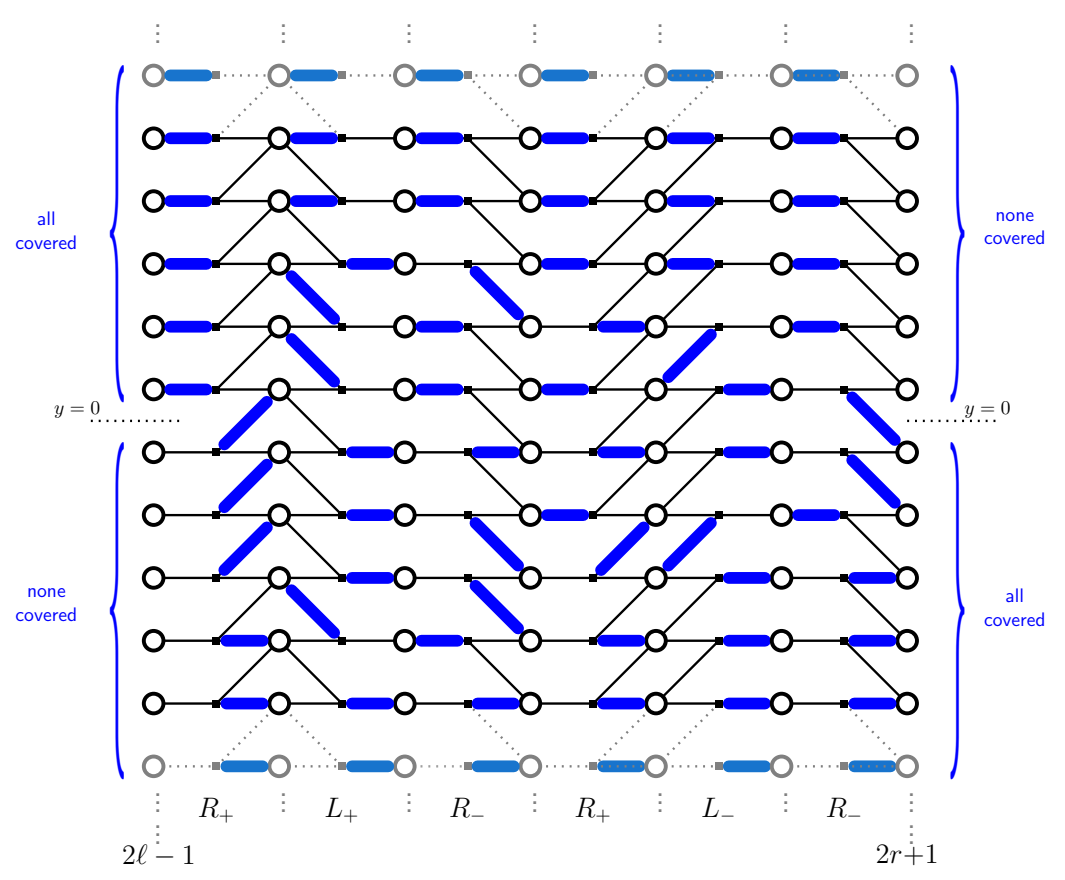

Figure 4. A pure dimer covering of the rail yard graph of Figure 1 .

domino tilings of the Aztec diamond of size $n$. We also recover plane partitions and so-called pyramid partitions, which requires taking $\ell=-\infty$ and $r=+\infty$ : plane partitions are obtained by taking a constant LR sequence and a sign sequence of the form $\cdots++++----\cdots$, while pyramid partitions are obtained by taking an alternating LR sequence $(\cdots L R L R L R L R \cdots)$ and the same sign sequence. We will discuss these specializations in greater detail in Section 5

2.3. Flips. We now define a local transformation on admissible coverings called the flip. Let $G$ be a rail yard graph, $C$ be an admissible covering of $G$, and let $f$ be an inner face of $G$. If exactly half of the edges bordering $f$ belong to $C$, then removing these edges from $C$ and replacing them by the other edges bordering $f$ gives another admissible covering $C^{\prime}$ of $G$. The operation that replaces $C$ by $C^{\prime}$ is called the flip of the face $f$, see Figure 5 .
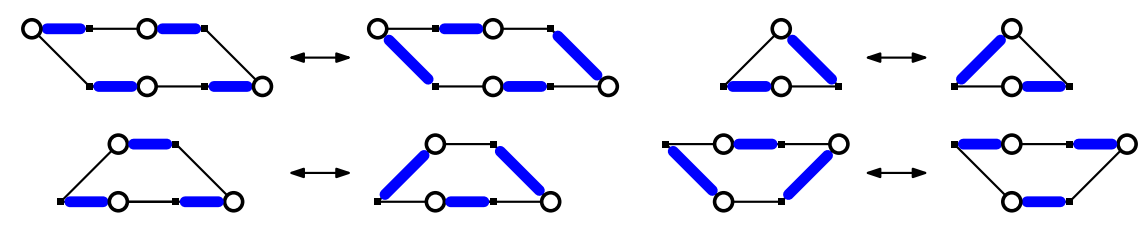

Figure 5. Examples of flips. All these flips are positive when the transformation is made from left to right.

We say that the flip of an inner face $f$ is positive if after performing the flip, the edges of $f$ that belong to the covering are oriented from odd to even vertices in counterclockwise direction around $f$. The flip is negative otherwise. For example, each flip displayed on Figure 5 is positive when performed from left to right. It follows from [Pro02, Theorem 2] that the positive flip relation endows the set of all pure coverings of a given rail yard graph with a distributive lattice structure. In 
particular, each rail yard graph has a unique minimal pure covering from which all other ones can be reached using positive flips only. The minimal covering is the only pure covering on which no negative flip is possible. Using this criterion one easily checks that the minimal covering coincides with the fundamental covering defined above. The flip distance between two coverings is the minimal number of flips needed to go from one to the other. When one of the two coverings is the fundamental one, the flip distance is realized by a sequence that uses positive flips only.

2.4. Enumeration. Our main enumerative result is an expression for the partition function of the RYG dimer model, which we now define. Consider a rail yard graph $G=\operatorname{RYG}(\ell, r, \underline{a}, \underline{b})$, and a sequence of formal variables $\underline{x}=\left(x_{\ell}, x_{\ell+1}, \ldots, x_{r}\right)$, where possibly $\ell=-\infty$ or $r=+\infty$. The weight of an admissible dimer covering $C$ of $G$ is then defined as

$$
w(C)=\prod_{i=\ell}^{r} x_{i}^{d_{i}(C)}
$$

where $d_{i}(C)$ is the number of diagonal dimers in column $i$ (i.e. the number of covered diagonal edges incident to an even vertex with abscissa $2 i$ ). This weight is welldefined since $\sum d_{i}(C)$ is finite by the definition of an admissible dimer covering. The partition function of the multivariate $R Y G$ dimer model, denoted $Z(G ; \underline{x})$, is then the sum of the weights of all pure dimer coverings of $G$.

Theorem 1. The partition function of the multivariate $R Y G$ dimer model reads

$$
Z(G ; \underline{x})=\prod_{\substack{\ell \leq i<j \leq r \\ b_{i}=+, b_{j}=-}} z_{i j}
$$

where

$$
z_{i j}= \begin{cases}1+x_{i} x_{j} & \text { if } a_{i} \neq a_{j}, \\ \left(1-x_{i} x_{j}\right)^{-1} & \text { if } a_{i}=a_{j} .\end{cases}
$$

Remark 2. The partition function is always a well-defined power series in the $x_{i}$ 's: indeed, all but finitely many factors contribute a factor 1 to the coefficient of a given monomial in 2 .

An interesting specialization is the $q-R Y G$ dimer model: given a formal variable $q$, we attach to each configuration a weight $q^{d}$ with $d$ its flip distance to the fundamental one. As explained in Section 3.3 below, this can be achieved by taking, for all $i \in[\ell . . r], x_{i}=q^{i}$ if $b_{i}=-$, and $x_{i}=1 / q^{i}$ if $b_{i}=+$, with $q$ an indeterminate. A caveat is that, when $\ell$ or $r$ is infinite, this specialization may be ill-defined since an infinite number of monomials in the $x_{i}$ 's might specialize to the same monomial $q^{d}$. A sufficient condition for the specialization to be well-defined is the following finiteness condition on the sign sequence:

- if $\ell=-\infty$, then there exists $\ell^{\prime}$ finite such that $b_{i}=+$ for all $i<\ell^{\prime}$,

- if $r=+\infty$, then there exists $r^{\prime}$ finite such that $b_{i}=-$ for all $i>r^{\prime}$.

(This condition is essentially necessary, because any initial run of - or final run of + in the sign sequence does not contribute to the partition function, and can be removed without loss of generality: any pure dimer covering coincides with the fundamental dimer covering in the corresponding regions.) 
Theorem 3. Assuming that the finiteness condition holds, the partition function of the $q-R Y G$ dimer model is

$$
\begin{gathered}
Z(G ; q)=\prod_{\substack{\ell \leq i<j \leq r \\
b_{i}=+, b_{j}=-}} z_{i j} \\
z_{i j}= \begin{cases}1+q^{j-i} & \text { if } a_{i} \neq a_{j}, \\
\left(1-q^{j-i}\right)^{-1} & \text { if } a_{i}=a_{j} .\end{cases}
\end{gathered}
$$

The proofs of Theorems 1 and 3 are given in Section 3.3

Remark 4. The product form (4) is strongly reminiscent of a hook-length formula, upon interpreting the sign sequence as describing the shape of a (possibly infinite) Young diagram, see Figure 6. The finiteness condition ensures that there are finitely many "hooks" of a given length, and hence that (4) is a well-defined formal power series in $q$.

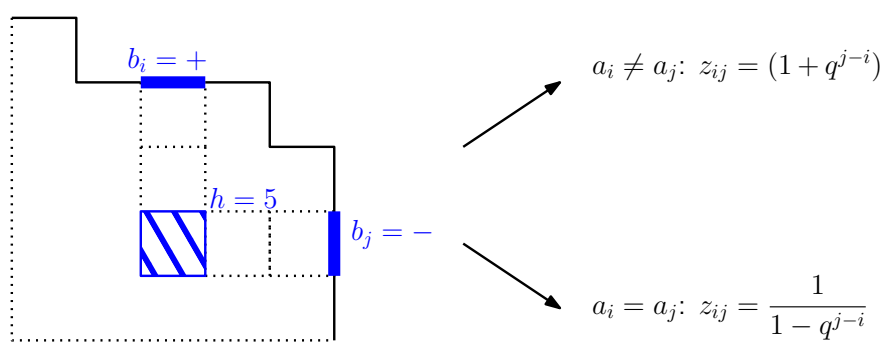

FiguRE 6. The interpretation of Theorem 3 as a hook-length formula. Displaying the sign sequence $\underline{b}$ as a lattice path whose horizontal (resp. vertical) steps correspond to + (resp. to - ), one obtains a Young diagram whose boxes are indexed by pairs $(i, j)$ such that $i<j, b_{i}=+, b_{j}=-$. The quantity $h=j-i$ is the "hook-length" of the box. In (4), each box gives rise to a multiplicative factor $z_{i, j}$ whose value $1+q^{h}$ or $\left(1-q^{h}\right)^{-1}$ is determined by comparing the two terms $a_{i}$ and $a_{j}$ of the LR sequence $\underline{a}$.

2.5. Correlations. So far we have introduced the partition function of the RYG dimer model, which depends on a sequence of formal variables $\underline{x}$ in general and on a single variable $q$ in the flip specialization. For a probabilistic or statistical physics interpretation, one shall rather consider the $x_{i}$ 's or $q$ as nonnegative real numbers such that the sum $Z(G ; \underline{x})$ of the weights $w(C)$ over all pure dimer coverings $C$ is convergent. As apparent from Theorem 11, this is the case if and only if

$$
x_{i} x_{j}<1 \text { for all } i<j \text { such that } a_{i}=a_{j}, b_{i}=+ \text { and } b_{j}=-
$$

and, when $\ell$ or $r$ is infinite,

$$
\sum_{\substack{\ell \leq i<j \leq r \\ b_{i}=+, b_{j}=-}} x_{i} x_{j}<\infty .
$$

In the $q$-RYG dimer model, (6) is satisfied whenever $q<1$, and (7) amounts to the finiteness condition defined before.

Assuming that the RYG dimer model is well-defined, that is to say (6) and (7) are satisfied, we may interpret $w(C) / Z(G ; \underline{x})$ as the probability of the pure dimer covering $C$. For a finite set $E$ of edges, we denote by $P_{G ; \underline{x}}(E)$ the probability 
that all the edges of $E$ are covered by a dimer. Our main probabilistic result is an explicit determinantal expression for $P_{G ; \underline{x}}(E)$, which requires to introduce some notations. For $k, k^{\prime}$ two integers, we set

$$
F_{k}(z)=\frac{\prod_{\substack{i:\left(a_{i}, b_{i}\right)=(R,+) \\ 2 i<k}}\left(1+x_{i} z\right) \prod_{\substack{i:\left(a_{j}, b_{j}\right)=(L,-) \\ 2 j>k}}\left(1-\frac{x_{j}}{z}\right)}{\prod_{\substack{\left.i, a_{i}\right)=(L,+) \\ 2 i \leq k}}\left(1-x_{i} z\right) \prod_{\substack{j:\left(a_{j}, b_{j}\right)=(R,-) \\ 2 j \geq k}}\left(1+\frac{x_{j}}{z}\right)} .
$$

and

$$
G_{k, k^{\prime}}(z, w)=\frac{F_{k}(z)}{F_{k^{\prime}}(w)} \frac{\sqrt{z w}}{z-w} .
$$

Note that all the products in (8) are convergent by (7), hence $F_{k}(z)$ is a meromorphic function on the whole complex plane, whose all zeros and poles are on the real axis.

For $\alpha, \beta$ two vertices of $G$ such that $\alpha^{\mathrm{x}}$ is even and $\beta^{\mathrm{x}}$ is odd, we set

$$
\mathcal{C}_{\alpha, \beta}=\frac{1}{(2 i \pi)^{2}} \oint_{C_{z}} \oint_{C_{w}} G_{\alpha^{\mathrm{x}}, \beta^{\mathrm{x}}}(z, w) \frac{w^{\beta^{\mathrm{y}}}}{z^{\alpha^{\mathrm{y}}}} \frac{\mathrm{d} z}{z} \frac{\mathrm{d} w}{w}
$$

where the contours must satisfy the following conditions: (i) $C_{z}$ should encircle 0 and all the negative poles of $F_{\alpha^{\mathrm{x}}}(z)$, but not the positive ones; (ii) $C_{w}$ should encircle 0 and all the positive zeros of $F_{\beta^{\mathrm{x}}}(w)$, but not the negative ones; (iii) $C_{z}$ and $C_{w}$ should not intersect, and $C_{z}$ should surround $C_{w}$ if and only if $\alpha^{\mathrm{x}}<\beta^{\mathrm{x}}$. We shall check in Section 4.4 that the assumptions (6) and (7) imply that such contours always exist but, at this stage, let us mention their intuitive interpretation: $\mathcal{C}_{\alpha, \beta}$ is obtained by extracting the coefficient of $z^{\alpha^{y}} w^{-\beta^{y}}$ in $G_{\alpha^{\mathrm{x}}, \beta^{\mathrm{x}}}(z, w)$, when we treat each factor $\left(1-x_{i} z\right)^{-1}$ as a power series in $z$, each factor $\left(1+x_{j} / z\right)^{-1}$ as a power series in $z^{-1}$, each factor $\left(1+x_{i} w\right)^{-1}$ as a power series in $w$, each factor $\left(1-x_{j} / w\right)^{-1}$ as a power series in $w^{-1}$, and finally we expand $\sqrt{z w} /(z-w)=\sum_{k \in \mathbb{N}+1 / 2}(w / z)^{k}$ if $\alpha^{\mathrm{x}}<\beta^{\mathrm{x}}$, or $\sqrt{z w} /(z-w)=\sum_{k \in \mathbb{N}+1 / 2}(w / z)^{-k}$ if $\alpha^{\mathrm{x}}>\beta^{\mathrm{x}}$.

We are now ready to state our theorem. Recall that, for an edge $e=(\alpha, \beta), \alpha$ and $\beta$ are assumed to be respectively the even and the odd endpoint of $e$.

Theorem 5 (Dimer correlations). Let $E=\left\{e_{1}, \ldots, e_{s}\right\}$ be a finite set of edges of $\operatorname{RYG}(\ell, r, \underline{a}, \underline{b})$, with $e_{i}=\left(\alpha_{i}, \beta_{i}\right)$. Then, we have

$$
P_{G ; \underline{x}}(E)=(-1)^{H(E)} \underline{x}^{\underline{n}} \operatorname{det}_{1 \leq i, j \leq s}\left(\mathcal{C}_{\alpha_{i}, \beta_{j}}\right),
$$

with $H(E)$ the number of horizontal edges in $E$ whose right endpoint is at an even abscissa, $\underline{x}^{\underline{n}}=x_{\ell}^{n_{\ell}} \cdots x_{r}^{n_{r}}$ with $n_{k}$ the number of diagonal edges in $E$ in column $k$, and $\mathcal{C}$ defined as in 10 .

The proof of Theorem 5 is given in Section 4 , where we also prove that the infinite matrix $\mathcal{C}$, with rows indexed by even vertices $\alpha$ and columns by odd vertices $\beta$, is an inverse of the Kasteleyn matrix of the rail yard graph for a suitable Kasteleyn orientation (see Theorem 17). Applications will be discussed in Section 5

\section{BOSONIC OPERATORS}

The purpose of this section is to establish Theorems 1 and 3 . This is done naturally by the transfer-matrix method, which here consists in decomposing the pure dimer coverings we want to enumerate into a sequence of compatible elementary dimer coverings. It turns out that the transfer matrices are isomorphic to certain 
operators arising in the so-called boson-fermion correspondence. For more details on this latter subject, we refer the reader to one of the many references available in the mathematical physics literature, for instance [Kac90, Chapter 14], MJD00, Oko01, Appendix A], OR07, Tin11 and [AZ13.

We start by giving the necessary reminders in Section 3.1, then make the connection with rail yard graphs in Section 3.2. and finally complete the proofs of Theorems 1 and 3 in Section 3.3 .

3.1. Reminders. An integer partition, or partition for short, is a nonincreasing sequence $\lambda=\left(\lambda_{i}\right)_{i \geq 1}$ of integers which vanishes eventually. The size of a partition $\lambda$ is $|\lambda|=\sum_{i \geq 1} \lambda_{i}$. We say that two partitions $\lambda$ and $\mu$ are interlaced, and we write $\lambda \succ \mu$ or $\mu \prec \lambda$, if we have

$$
\lambda_{1} \geq \mu_{1} \geq \lambda_{2} \geq \mu_{2} \geq \lambda_{3} \geq \cdots
$$

In the well-known pictorial representation in terms of Young diagrams, this means that the skew shape $\lambda / \mu$ is a horizontal strip, see e.g. [Sta99, Chap. 7] for more precise definitions. To a partition $\lambda$ we may associate its conjugate $\lambda^{\prime}$, whose Young diagram is the image of that of $\lambda$ by a reflection along the main diagonal. In more explicit terms, we have $\lambda_{i}^{\prime}=\#\left\{j \geq 0, \lambda_{j} \geq i\right\}$. Note that the relation $\lambda \succ \mu$ amounts to

$$
\lambda_{i}^{\prime}-\mu_{i}^{\prime} \in\{0,1\} \quad \text { for all } i \geq 1 .
$$

The bosonic Fock space, denoted $\mathcal{B}$, is the infinite dimensional Hilbert space spanned by orthonormal basis vectors $|\lambda\rangle$ where $\lambda$ runs over the set of integer partitions. Here we will use the bra-ket notation so that $\langle\lambda|$ denotes the dual basis vector. For $x$ a formal or complex variable, we introduce the operators $\Gamma_{L+}(x), \Gamma_{L-}(x), \Gamma_{R+}(x), \Gamma_{R-}(x)$ whose action on basis vectors reads

$$
\begin{aligned}
\Gamma_{L+}(x)|\lambda\rangle & =\sum_{\mu: \mu \prec \lambda} x^{|\lambda|-|\mu|}|\mu\rangle, & \Gamma_{R+}(x)|\lambda\rangle & =\sum_{\mu: \mu^{\prime} \prec \lambda^{\prime}} x^{|\lambda|-|\mu|}|\mu\rangle, \\
\Gamma_{L-}(x)|\lambda\rangle & =\sum_{\mu: \mu \succ \lambda} x^{|\mu|-|\lambda|}|\mu\rangle, & & \Gamma_{R-}(x)|\lambda\rangle=\sum_{\mu: \mu^{\prime} \succ \lambda^{\prime}} x^{|\mu|-|\lambda|}|\mu\rangle .
\end{aligned}
$$

These operators are sometimes called (half-)vertex operators. Let us mention that, in the literature, $\Gamma_{L \pm}(x)$ is often denoted $\Gamma_{ \pm}(x)$, see e.g. Oko01, while $\Gamma_{R \pm}(x)$ is sometimes denoted $\Gamma_{ \pm}^{\prime}(x)$ You10, BCC14]. Observe that we have

$$
\Gamma_{L+}(x)|\emptyset\rangle=\Gamma_{R+}(x)|\emptyset\rangle=|\emptyset\rangle, \quad\langle\emptyset| \Gamma_{L-}(x)=\langle\emptyset| \Gamma_{R-}(x)=\langle\emptyset|
$$

where $\emptyset$ denotes the empty partition. Note also that $\Gamma_{L-}\left(\operatorname{resp} . \Gamma_{R-}\right)$ is the dual of $\Gamma_{L+}\left(\right.$ resp. $\left.\Gamma_{R+}\right)$, and that $\Gamma_{R+}\left(\operatorname{resp} . \Gamma_{R-}\right)$ is conjugated to $\Gamma_{L+}\left(\operatorname{resp} . \Gamma_{L-}\right)$ via the involution $\omega$ of $\mathcal{B}$ sending $|\lambda\rangle$ to $\left|\lambda^{\prime}\right\rangle$.

Remark 6. For $a_{1}, a_{2} \in\{L, R\}$, the product $\Gamma_{a_{1}-}\left(x_{1}\right) \Gamma_{a_{2}+}\left(x_{2}\right)$ is clearly welldefined, because its coefficient between two states $\langle\lambda|$ and $|\mu\rangle$ involves only a finite sum. The same is true for $\Gamma_{a_{1}+}\left(x_{1}\right) \Gamma_{a_{2}-}\left(x_{2}\right)$ when $a_{1} \neq a_{2}$ (observe that the "intermediate" partitions cannot get too large). Infinite sums arise when considering $\Gamma_{a_{1}+}\left(x_{1}\right) \Gamma_{a_{2}-}\left(x_{2}\right)$ with $a_{1}=a_{2}$, but its coefficients are power series in $x_{1}$ and $x_{2}$, which are convergent for $\left|x_{1} x_{2}\right|<1$ as apparent from the following proposition.

Proposition 7 (Commutation relations). For $a_{1}, a_{2} \in\{L, R\}$, we have

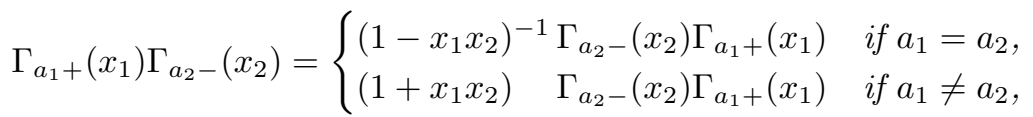

while $\Gamma_{a_{1}+}\left(x_{1}\right)$ commutes with $\Gamma_{a_{2}+}\left(x_{2}\right)$, and $\Gamma_{a_{1}-}\left(x_{1}\right)$ commutes with $\Gamma_{a_{2}-}\left(x_{2}\right)$. 
Proof. See for instance You10, Lemma 3.3] for an algebraic proof, and $\mathrm{BBB}^{+} 14$, Section 3] for a bijective proof of (16). The celebrated Bender-Knuth involution BK72, pp. 46-47] implies that $\Gamma_{a \pm}\left(x_{1}\right)$ commutes with $\Gamma_{a \pm}\left(x_{2}\right)$ for $a=L$ or $R$. That $\Gamma_{L \pm}\left(x_{1}\right)$ commutes with $\Gamma_{R \pm}\left(x_{2}\right)$ is also well-known, but for completeness let us here sketch a short proof: for two partitions $\lambda, \mu$, one sees easily that the two sets $\left\{\nu: \mu \prec \nu, \nu^{\prime} \prec \lambda^{\prime}\right\}$ and $\left\{\nu: \mu^{\prime} \prec \nu^{\prime}, \nu \prec \lambda\right\}$ are nonempty if and only if $\lambda / \mu$ is a skew shape containing no $2 \times 2$ square. In that case, both sets have the same cardinality $2^{C}$, where $C$ is the number of connected components of $\lambda / \mu$, and one easily constructs a bijection between them proving the wanted commutation relation. Another byproduct of this bijection is that

$$
\Gamma_{L \pm}(x) \Gamma_{R \pm}(-x)=\Gamma_{R \pm}(-x) \Gamma_{L \pm}(x)=1 .
$$

Given two symbols • and o (called respectively black and white marbles), a Maya diagram [MJD00] is an element $\mathbf{m}$ of $\{\bullet, \circ\}^{\mathbb{Z}+1 / 2}$ such that $\mathbf{m}_{k}$ is eventually equal to $\bullet$ for $k \rightarrow-\infty$ and to $\circ$ for $k \rightarrow+\infty$. It then not difficult to check that the quantity

$$
c=\#\left\{k>0, \mathbf{m}_{k}=\bullet\right\}-\#\left\{k<0, \mathbf{m}_{k}=\circ\right\}
$$

is a finite integer, and we call it the charge of $\mathbf{m}$. Let $k_{1}>k_{2}>\cdots$ be the positions of $\bullet$ in $\mathbf{m}$ enumerated in decreasing order, and let $\lambda_{i}=k_{i}-c+i-1 / 2$ : it is easily seen that $\lambda$ is a partition and that the correspondence $\mathbf{m} \mapsto(\lambda, c)$ is one-to-one, the pair $(\lambda, c)$ being called a charged partition. Observe that we may extend the involution $\omega$ to charged partitions by setting $\omega(\lambda, c)=\left(\lambda^{\prime},-c\right)$, and this corresponds on Maya diagrams to performing a reflection across 0 and exchanging - and $\circ$ : in other words, $\mathbf{m}$ is sent to $\mathbf{m}^{\prime}$ such that $\left\{\mathbf{m}_{k}, \mathbf{m}_{-k}^{\prime}\right\}=\{\bullet, \circ\}$ for all $k \in \mathbb{Z}+1 / 2$. By a slight abuse, we still denote by $\emptyset$ the Maya diagram of charge 0 corresponding to the empty partition.

The fermionic Fock space, denoted $\mathcal{F}$, is the infinite dimensional Hilbert space spanned by orthonormal basis vectors $|\mathbf{m}\rangle$ where $\mathbf{m}$ runs over the set of all Maya diagrams. For $c \in \mathbb{Z}$, let $\mathcal{F}_{c} \subset \mathcal{F}$ denote the subspace spanned by Maya diagrams of charge $c$, so that $\mathcal{F}=\oplus_{c \in \mathbb{Z}} \mathcal{F}_{c}$. By the bijection between Maya diagrams and charged partitions, each $\mathcal{F}_{c}$ may be canonically identified with $\mathcal{B}$. This defines the action of the bosonic operators $\Gamma_{L \pm}$ and $\Gamma_{R \pm}$ on $\mathcal{F}$, leaving each subspace $\mathcal{F}_{c}$ invariant (by a slight abuse we keep the same notations for the operators acting on this larger space, and note that the commutations relations of Proposition 7 remain valid). We now end this section devoted to reminders, leaving the discussion of fermionic operators to Section 4.1 .

3.2. Interpretation as transfer matrices for RYGs. The purpose of this section is to explain how the operators $\Gamma_{L \pm} / \Gamma_{R \pm}$ may be identified with dimer transfer matrices. The key observation is that Maya diagrams describe the boundary states in our model. More precisely, let us consider an admissible dimer covering $C$ of $G=\operatorname{RYG}(\ell, r, \underline{a}, \underline{b})$. We define the left boundary state $\mathrm{l}(C)$ of $C$ by setting, for all $k \in \mathbb{Z}+1 / 2$,

$$
\mathbf{l}(C)_{k}= \begin{cases}\circ & \text { if }(2 \ell-1, k) \text { is covered by a dimer, } \\ \bullet & \text { otherwise. }\end{cases}
$$

It is a Maya diagram by the definition of an admissible dimer covering. Similarly, the right boundary state $\mathbf{r}(C)$ of $C$ is the Maya diagram defined by

$$
\mathbf{r}(C)_{k}= \begin{cases}\bullet & \text { if }(2 \ell+1, k) \text { is covered by a dimer } \\ \circ & \text { otherwise }\end{cases}
$$


for all $k \in \mathbb{Z}+1 / 2$. See Figure 7 (a). A pure dimer covering has both boundary states equal to $\emptyset$. Note that, if $G^{\prime}$ is a rail yard graph which is concatenable after $G$, and if $C^{\prime}$ is an admissible dimer covering of $G^{\prime}$, then $C$ and $C^{\prime}$ are compatible if and only if $\mathbf{r}(C)=\mathbf{l}\left(C^{\prime}\right)$. We may now state the main result of this section.

Proposition 8 (Transfer matrix decomposition). Given a rail yard graph $G=$ $\operatorname{RYG}(\ell, r, \underline{a}, \underline{b})$ with $\ell, r$ finite, and two Maya diagrams $\mathbf{l}$ and $\mathbf{r}$, the sum of the weights (1) of all admissible dimer coverings of $G$ with left boundary state 1 and right boundary state $\mathbf{r}$ is given by

$$
Z(G, \mathbf{l}, \mathbf{r} ; \underline{x})=\left\langle\mathbf{l}\left|\Gamma_{a_{\ell} b_{\ell}}\left(x_{\ell}\right) \Gamma_{a_{\ell+1} b_{\ell+1}}\left(x_{\ell+1}\right) \cdots \Gamma_{a_{r} b_{r}}\left(x_{r}\right)\right| \mathbf{r}\right\rangle .
$$

In particular, the partition function reads

$$
Z(G ; \underline{x})=\left\langle\emptyset\left|\Gamma_{a_{\ell} b_{\ell}}\left(x_{\ell}\right) \Gamma_{a_{\ell+1} b_{\ell+1}}\left(x_{\ell+1}\right) \cdots \Gamma_{a_{r} b_{r}}\left(x_{r}\right)\right| \emptyset\right\rangle .
$$

Proof. Note that (22) follows from (21) by taking $\mathbf{l}=\mathbf{r}=\emptyset$, which amounts to considering pure dimer coverings.

We first verify (21) for $\ell=r$, i.e. when $G$ is an elementary rail yard graph. Let us here treat the case $a_{\ell}=L, b_{\ell}=+$ (displayed third on Figure2(c)) and leave the other cases to the reader. Let $s_{1}<s_{2}<\cdots\left(\right.$ resp. $\left.t_{1}<t_{2}<\cdots\right)$ be the positions of $\circ$ in $\mathbf{l}$ (resp. $\mathbf{r}$ ) enumerated in increasing order. Then, we claim that both sides of (21) are equal to $x_{\ell}^{\sum\left(s_{i}-t_{i}\right)}$ if the two conditions

$$
\begin{gathered}
s_{i}-t_{i} \in\{0,1\} \quad \text { for all } i \geq 1 \\
\sum_{i \geq 1}\left(s_{i}-t_{i}\right)<\infty
\end{gathered}
$$

hold, and that both sides vanish otherwise. Indeed, on the one hand, it is not difficult to check (see Figure 7) that there is at most one elementary dimer configuration with prescribed boundary states $\mathbf{l}$ and $\mathbf{r}$, and that there is exactly one such configuration (containing $\sum\left(s_{i}-t_{i}\right)$ diagonal dimers) if and only if (23) and (24) hold. On the other hand, let $\lambda$ and $\mu$ be the integer partitions associated with the Maya diagrams $\mathbf{l}$ and $\mathbf{r}$ : the quantity $\left\langle\mathbf{l}\left|\Gamma_{L+}\left(x_{\ell}\right)\right| \mathbf{r}\right\rangle$ is equal to $x_{\ell}^{|\mu|-|\lambda|}$ if the two conditions

$$
\lambda \prec \mu
$$

$\mathbf{l}$ and $\mathbf{r}$ have the same charge $c$

hold, and it vanishes otherwise. But we have $\lambda_{i}^{\prime}=c+i-1 / 2-s_{i}$ and $\mu_{i}^{\prime}=$ $c+i-1 / 2-t_{i}$ for all $i \geq 1$ hence, in view of $(13)$, we find that the conditions (23)-(24) amount to $(25)-(\sqrt{26})$, and then that $|\mu|-|\lambda|=\sum\left(s_{i}-t_{i}\right)$ as wanted.

It remains to verify (21) for $\ell<r$, which may be easily done by induction: it suffices to observe that, if $G^{\prime}$ is the rail yard graph obtained by removing the last "strip" of $G$, then any admissible dimer covering $C$ of $G$ with boundary states $\mathbf{l}, \mathbf{r}$ is uniquely decomposed into a pair formed by an admissible dimer covering $C^{\prime}$ of $G^{\prime}$ with boundary states $\mathbf{l}, \mathbf{m}$, for some Maya diagram $\mathbf{m}$, and an elementary dimer covering $E$ with boundary states $\mathbf{m}, \mathbf{r}$, such that $w(C)=w\left(C^{\prime}\right) w(E)$.

3.3. Proof of enumeration results and computation of the partition function. We are now ready to prove Theorems 1 and 3. The first one is a direct consequence of the formalism developed above, whereas the second deserves an inspection of the different types of flips in rail yard graphs.

Proof of Theorem 1. We simply have to evaluate the right-hand side of (22), which can be done as in in [OR07, Section 4.1], You10, Section 4] or [BCC14, Section 


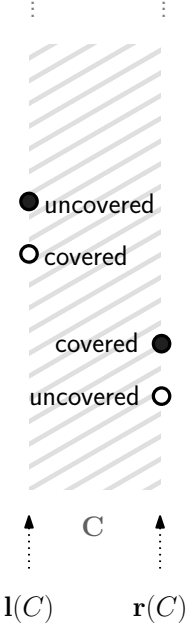

(a)

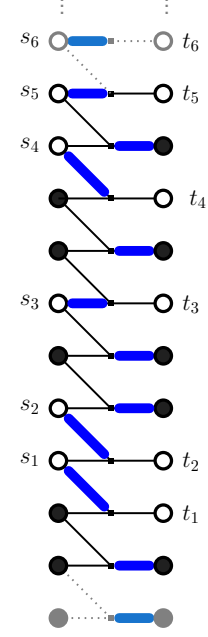

(b)
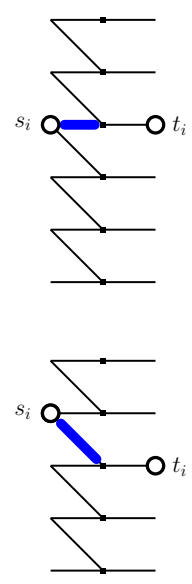

(c)

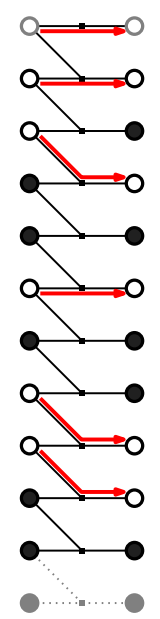

$(d)$

Figure 7. (a) The rules (19)- 20 that define the two Maya diagrams $\mathbf{l}(C)$ and $\mathbf{r}(C)$. (b) An admissible dimer covering of the elementary rail yard graph $L+$. White marbles are numbered as in the proof of Proposition 8. (c) If the left boundary vertex at ordinate $s_{i}$ is covered by a horizontal (resp. diagonal) dimer, then the right boundary vertex at ordinate $s_{i}$ (resp. $s_{i}-1$ ) is necessarily uncovered. Therefore there is a white marble at this ordinate on the right boundary, and induction implies that it is the $i$-th white marble on the right boundary. This proves that $s_{i}-t_{i} \in\{0,1\}$ and that $\sum_{i}\left(s_{i}-t_{i}\right)$ is equal to the number of diagonal dimers. Moreover, since dimers incident to the left boundary can be recovered from the knowledge of the sequences $\left(s_{i}\right)$ and $\left(t_{i}\right)$, it is clear that there is at most one dimer configuration with given boundary states $\mathbf{l}$ and $\mathbf{r}$, and that there is one if and only if (23) and 24) hold. (d) Condition (23) can be interpreted by saying that white marbles are "jumping downwards" by 0 or 1 . The cases of the elementary graphs $L-, R+$, and $R$ - have similar interpretation, respectively with white marbles jumping upwards, black marbles jumping upwards, and black marbles jumping downwards, in all cases by 0 or 1 .

5.1]: first observe that, by (15), for any $k, m$, any $\left(c_{1}, c_{2}, \ldots c_{k+m}\right) \in\{L, R\}^{k+m}$, one has:

$$
\left\langle\emptyset\left|\prod_{i=1}^{k} \Gamma_{c_{i}-}\left(z_{i}\right) \prod_{i=k+1}^{k+m} \Gamma_{c_{i}+}\left(z_{i}\right)\right| \emptyset\right\rangle=1,
$$

the $z_{i}$ being formal variables.

Now, by applying successively the commutation relations of Proposition 7, one can transform 22 into a scalar product of this form, up to a multiplicative prefactor, by moving to the left all the operators $\Gamma_{a_{j} b_{j}}\left(x_{j}\right)$ such that $b_{j}=-$. In order to do that, we have, for each $\ell \leq i<j \leq r$ such that $b_{i}=+$ and $b_{j}=-$, to transform the product of operators $\Gamma_{a_{i}+}\left(x_{i}\right) \Gamma_{b_{j}-}\left(x_{j}\right)$ into the product $\Gamma_{b_{j}-}\left(x_{j}\right) \Gamma_{a_{i}+}\left(x_{i}\right)$. For each such transformation, we obtain a multiplicative contribution given by $(16)$, and the result follows. 
Proof of Theorem 3. As explained before the statement of Theorem 3, we have to prove that, specializing for $i \in[l . . r]$ the weights (1) to $x_{i}=q^{i}$ if $b_{i}=-$ and $x_{i}=1 / q^{i}$ if $b_{i}=+$ amounts to attaching to each configuration a weight $q^{d}$, where $d$ is the flip distance to the fundamental configuration.

First, this is true for the fundamental configuration that receives a weight 1 in both cases. Second, since by Propp's theory (recalled in Section 2.3) each shortest path from the fundamental state to any configuration is realized using positive flips only, it is enough to check that, in this specialization, each positive flip increases the weight of a configuration by a factor of $q$.
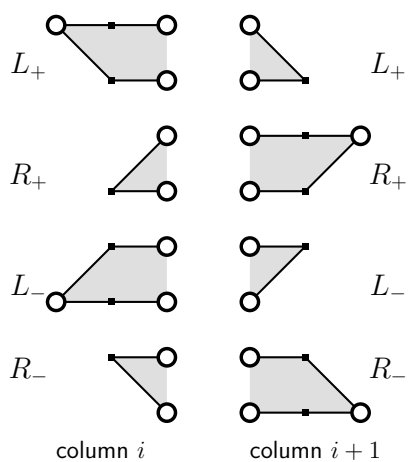

(a)

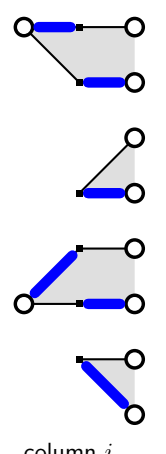

(b)
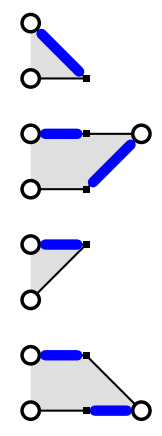

column $i+1$

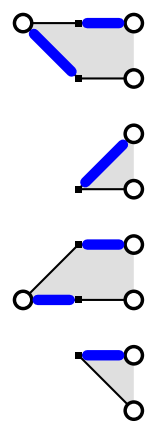

column $i$ (c)

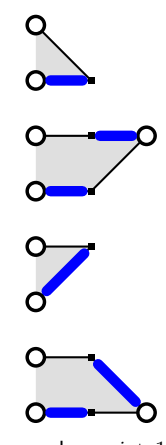

column $i+1$

Figure 8. (a) The 16 possible face types of rail yard graphs are obtained by matching one of the half-face types on the left with one on the right. (b-c) The dimer configuration around half-faces before (b) and after (c) a positive flip.

Consider an inner face $f$ in a rail yard graph. Then $f$ is made by the union of two half-faces as shown on Figure 8(a). Each of these two half-faces is incident to a diagonal edge, one in column $i$, and one in column $i+1$, in the sense of Section 2.4. for some $i \in[l . . r-1]$. Then, a case inspection (see Figure $8(\mathrm{~b}-\mathrm{c})$ ) shows that the following is true: when performing a positive flip on $f$, the number of diagonal dimers on column $i$ increases (resp. decreases) by 1 if $b_{i}=+\left(\right.$ resp. $b_{i}=-$ ), and the number of diagonal dimers on column $i+1$ decreases (resp. increases) by 1 if $b_{i+1}=+$ (resp. $\left.b_{i+1}=-\right)$.

Therefore this flip multiplies the weight (1) of the configuration by a factor of:

$$
x_{i}^{b_{i}} / x_{i+1}^{b_{i+1}}
$$

where we identified,+- with $+1,-1$ respectively. But, in the specialization we are considering, we have $x_{j}^{b_{j}}=q^{-j}$ for all $j \in[l . . r]$, so $[28)$ is equal to $q$ and the proof is complete.

\section{FERMIONIC OPERATORS}

The purpose of this section is to establish Theorem 5. We start in Section 4.1 by recalling the definitions and basic properties of fermionic operators. In Section 4.2. we show that these operators can be used to construct constrained transfer matrices, that is transfer matrices enumerating dimer configurations containing a given subset of edges. We rewrite the product of constrained transfer matrices in another convenient form in Section 4.3, and complete the proof of Theorem 5 in Section 4.4 . Finally, we elucidate the connection with Kasteleyn's theory in Section 4.5. 
4.1. Reminders. Recall that the fermionic Fock space $\mathcal{F}$, introduced at the end of Section 3.1, is the infinite dimensional Hilbert space spanned by orthonormal basis vectors $|\mathbf{m}\rangle$ where $\mathbf{m}$ runs over the set of all Maya diagrams. For $k \in \mathbb{Z}+1 / 2$, we define the fermionic operators $\psi_{k}$ and $\psi_{k}^{*}$ (also called creation/annihilation operators) through their action on a basis vector $|\mathbf{m}\rangle$ by

$$
\begin{aligned}
& \psi_{k}|\mathbf{m}\rangle= \begin{cases}(-1)^{\#\left\{j>k, \mathbf{m}_{j}=\bullet\right.}\left|\mathbf{m}^{(k)}\right\rangle & \text { if } \mathbf{m}_{k}=0, \\
0 & \text { otherwise, }\end{cases} \\
& \psi_{k}^{*}|\mathbf{m}\rangle= \begin{cases}(-1)^{\#\left\{j>k, \mathbf{m}_{j}=\bullet\right\}}\left|\mathbf{m}^{(k)}\right\rangle & \text { if } \mathbf{m}_{k}=\bullet \\
0 & \text { otherwise },\end{cases}
\end{aligned}
$$

where $\mathbf{m}^{(k)}$ is the Maya diagram obtained from $\mathbf{m}$ by inverting the color of the marble on site $k$. Observe that the operators $\psi_{k}$ and $\psi_{k}^{*}$ are adjoint to one another. In particular, $\psi_{k} \psi_{k}^{*}$ (resp. $\psi_{k}^{*} \psi_{k}$ ) is the orthogonal projector on the space spanned by Maya diagrams $\mathbf{m}$ with $\mathbf{m}_{k}=\bullet$ (resp. $\mathbf{m}_{k}=0$ ). Fermionic operators obey the following well-known canonical anticommutation relations:

Proposition 9. For any $k$ and $k^{\prime}$ in $\mathbb{Z}+1 / 2$, we have

$$
\left\{\psi_{k}, \psi_{k^{\prime}}\right\}=0, \quad\left\{\psi_{k}^{*}, \psi_{k^{\prime}}^{*}\right\}=0, \quad\left\{\psi_{k}, \psi_{k^{\prime}}^{*}\right\}=\delta_{k, k^{\prime}}
$$

Here $\{a, b\}$ denotes the anticommutator of $a$ and $b:\{a, b\}=a b+b a$.

Proof. Easy.

Define the fermionic generating functions

$$
\psi(z)=\sum_{k \in \mathbb{Z}+\frac{1}{2}} z^{k} \psi_{k}, \quad \psi^{*}(z)=\sum_{k \in \mathbb{Z}+\frac{1}{2}} z^{-k} \psi_{k}^{*}
$$

Proposition 9 translates into

$$
\{\psi(z), \psi(w)\}=0, \quad\left\{\psi^{*}(z), \psi^{*}(w)\right\}=0, \quad\left\{\psi(z), \psi^{*}(w)\right\}=\delta(z, w)
$$

where $\delta(z, w)=\sum_{k \in \mathbb{Z}+\frac{1}{2}}(z / w)^{k}$ is the formal Dirac delta function. It is straightforward to check that

$$
\begin{aligned}
& \left\langle\emptyset\left|\psi(z) \psi^{*}(w)\right| \emptyset\right\rangle=\sum_{\substack{k \in \mathbb{Z}+1 / 2 \\
k<0}}\left(\frac{z}{w}\right)^{k}=\frac{\sqrt{z w}}{z-w} \quad \text { for }|z|>|w|, \\
& \left\langle\emptyset\left|\psi^{*}(w) \psi(z)\right| \emptyset\right\rangle=\sum_{\substack{k \in \mathbb{Z}+1 / 2 \\
k>0}}\left(\frac{z}{w}\right)^{k}=-\frac{\sqrt{z w}}{z-w} \text { for }|w|>|z| .
\end{aligned}
$$

Here the leftmost equal signs correspond to formal identities, but the rightmost equal signs require to treat $z$ and $w$ as complex variables. Let us also mention a lesser-known fact about the action of the involution $\omega$ on the fermionic operators (recall that $\omega$ is the involution that maps a charged partition $(\lambda, c)$ to $\left(\lambda^{\prime},-c\right)$, hence can be seen as acting on $\mathcal{F}$ ).

Proposition 10. For $k \in \mathbb{Z}+1 / 2$, we have

$$
\omega \psi_{k}^{*} \omega=(-1)^{C+k+1 / 2} \psi_{-k}, \quad \text { i.e. } \quad \omega \psi^{*}(z) \omega=(-1)^{C+1 / 2} \psi(-z)
$$

where $C$ is the charge operator (acting on $\mathcal{F}_{c}$ as the multiplication by $c$ ).

Proof. Follows from the fact that, for any integer $k^{\prime}$ and any Maya diagram $\mathbf{m}$ of charge $c$, we have

$$
\#\left\{j>k^{\prime}, \mathbf{m}_{j}=\bullet\right\}-\#\left\{j<k^{\prime}, \mathbf{m}_{j}=\circ\right\}=c-k^{\prime} .
$$


Last but not least, we have the following commutation relations between bosonic and fermionic operators.

Proposition 11. Given two formal variables $x, z$ we have

$$
\begin{aligned}
\Gamma_{L+}(x) \psi(z) & =\frac{1}{1-x z} \psi(z) \Gamma_{L+}(x) \\
\Gamma_{R+}(x) \psi(z) & =(1+x z) \psi(z) \Gamma_{R+}(x) \\
\Gamma_{L+}(x) \psi^{*}(z) & =(1-x z) \psi^{*}(z) \Gamma_{L+}(x) \\
\Gamma_{R+}(x) \psi^{*}(z) & =\frac{1}{1+x z} \psi^{*}(z) \Gamma_{R+}(x) \\
\psi(z) \Gamma_{L-}(x) & =(1-x / z) \Gamma_{L-}(x) \psi(z) \\
\psi(z) \Gamma_{R-}(x) & =\frac{1}{1+x / z} \Gamma_{R-}(x) \psi(z) \\
\psi^{*}(z) \Gamma_{L-}(x) & =\frac{1}{1-x / z} \Gamma_{L-}(x) \psi^{*}(z) \\
\psi^{*}(z) \Gamma_{R-}(x) & =(1+x / z) \Gamma_{R-}(x) \psi^{*}(z)
\end{aligned}
$$

The first four (resp. last four) formal identities correspond to actual converging series when $|z|<x^{-1}$ (resp. $|z|>x$ ).

We give in Appendix A a combinatorial proof of these identities (note that it is sufficient to establish only one of them, the others follow by taking duals, inverses and conjugates by $\omega$ ). For algebraic derivations, see for instance the references given at the beginning of Section 3 .

4.2. Constrained transfer matrices. The fermionic operators can be used to enumerate constrained dimer configurations. A first natural idea, already used in OR03, consists in inserting some orthogonal projectors $\psi_{k} \psi_{k}^{*}$ or $\psi_{k}^{*} \psi_{k}$ (with various $k$ 's) within the product of bosonic operators (22) forming the partition function, which has the effect of forcing black or white marbles to be present at given positions. However, this does not fully determine the positions of the dimers (there are ambiguities for the columns containing both horizontal and diagonal edges). Remarkably, for an arbitrary rail yard graph and an arbitrary finite set $E$ of edges, there is a suitable way of inserting fermionic operators which precisely forces each edge of $E$ to be covered by a dimer.

We first introduce convenient notations. Recall that writing $(\alpha, \beta)$ for an edge implies that its endpoints $\alpha$ and $\beta$ are such that $\alpha^{\mathrm{x}}$ is even and $\beta^{\mathrm{x}}$ is odd. Any finite set $E$ of edges of a rail yard graph can be decomposed "column by column", hence written in the form

$$
E=\bigcup_{i \in \mathbb{Z}}\left\{\left(\alpha_{i, 1}, \beta_{i, 1}\right), \ldots,\left(\alpha_{i, m_{i}}, \beta_{i, m_{i}}\right),\left(\gamma_{i, 1}, \delta_{i, 1}\right), \ldots,\left(\gamma_{i, m_{i}^{\prime}}, \delta_{i, m_{i}^{\prime}}\right)\right\}
$$

where $\alpha_{i, j}^{\mathrm{x}}=\gamma_{i, j}^{\mathrm{x}}=2 i, \beta_{i, j}^{\mathrm{x}}=2 i-1$ and $\delta_{i, j}^{\mathrm{x}}=2 i+1$. Here $m_{i}$ (resp. $\left.m_{i}^{\prime}\right)$ is the number of edges of $E$ connecting vertices with abscissas $2 i-1$ and $2 i$ (resp. $2 i$ and $2 i+1)$, and is zero except for finitely many $i$.

Theorem 12 (Constrained transfer matrix decomposition). Let $E$ be an arbitrary finite subset of edges of the graph $G=\operatorname{RYG}(\ell, r, \underline{a}, \underline{b})$, which we decompose as in (44), and let $n_{i}(i=\ell . . r)$ be the number of diagonal edges of $E$ with an endpoint of abscissa $2 i$. The sum of the weights (1) of all pure dimer configurations containing E reads

$$
W(G ; \underline{x}, E)=\left\langle\emptyset\left|T_{\ell} T_{\ell+1} \cdots T_{r}\right| \emptyset\right\rangle
$$


where, for all $i \in[\ell . . r]$, the constrained transfer matrix $T_{i}$ is given by

$$
T_{i}=(-1)^{k_{i}} x_{i}^{n_{i}}\left(\prod_{j=1}^{m_{i}} \psi_{\beta_{i, j}^{\mathrm{y}}}^{*}\right)\left(\prod_{j=1}^{m_{i}} \psi_{\alpha_{i, j}^{\mathrm{y}}}\right)\left(\prod_{j=1}^{m_{i}^{\prime}} \psi_{\gamma_{i, j}^{\mathrm{y}}}\right) \Gamma_{R, b_{i}}\left(x_{i}\right)\left(\prod_{j=1}^{m_{i}^{\prime}} \psi_{\delta_{i, j}^{\mathrm{y}}}^{*}\right)
$$

with $k_{i}=m_{i}\left(m_{i}-1\right) / 2+m_{i}^{\prime}\left(m_{i}^{\prime}-1\right) / 2$ if $a_{i}=R$, and

$$
T_{i}=(-1)^{k_{i}} x_{i}^{n_{i}}\left(\prod_{j=1}^{m_{i}} \psi_{\beta_{i, j}^{\mathrm{y}}}^{*}\right) \Gamma_{L, b_{i}}\left(x_{i}\right)\left(\prod_{j=1}^{m_{i}} \psi_{\alpha_{i, j}^{\mathrm{y}}}\right)\left(\prod_{j=1}^{m_{i}^{\prime}} \psi_{\gamma_{i, j}^{\mathrm{y}}}\right)\left(\prod_{j=1}^{m_{i}^{\prime}} \psi_{\delta_{i, j}^{\mathrm{y}}}^{*}\right)
$$

with $k_{i}=m_{i}\left(m_{i}-1\right) / 2+m_{i}^{\prime}\left(m_{i}^{\prime}-1\right) / 2+n_{i}$ if $a_{i}=L$. More generally, the sum of the weights of all admissible dimer coverings with left boundary state 1 and right boundary state $\mathbf{r}$ containing $E$ reads

$$
W(G, \mathbf{l}, \mathbf{r} ; \underline{x}, E)=\left\langle\mathbf{l}\left|T_{\ell} T_{\ell+1} \cdots T_{r}\right| \mathbf{r}\right\rangle .
$$

Observe that we recover $(22)$ and 21$]$ when $E$ is empty. The order in which we take the products of fermionic operators in 46 and (47) is irrelevant, as long as we take the same order for both products from 1 to $m_{i}$, and for both products from 1 to $m_{i}^{\prime}$ (otherwise, we might get a wrong sign).

Proof of Theorem 12. It is sufficient to prove (48) in the case of an elementary RYG, i.e. to prove that $T_{i}$ is indeed the wanted constrained transfer matrix. The general case immediately follows by concatenation (i.e. by the transfer matrix method), as done in the proof of Proposition 8 .

Let $G=\operatorname{RYG}\left(i, i, a_{i}, b_{i}\right)$ be an elementary RYG, $\mathbf{l}, \mathbf{r}$ two boundary states, and $E$ a finite subset of edges of $G$. The general decomposition 44 reads here simply

$$
E=\left\{\left(\alpha_{1}, \beta_{1}\right), \ldots,\left(\alpha_{m}, \beta_{m}\right),\left(\gamma_{1}, \delta_{1}\right), \ldots,\left(\gamma_{m^{\prime}}, \delta_{m^{\prime}}\right)\right\}
$$

Let us first assume that $a_{i}=R$, we then need to check that

$$
W\left(G, \mathbf{l}, \mathbf{r} ; x_{i}, E\right)=\left\langle\mathbf{l}\left|T_{i}\right| \mathbf{r}\right\rangle .
$$

with $T_{i}$ given by (46). This is immediate in the case $m^{\prime}=0$ : indeed the presence of a dimer on the edge $\left(\alpha_{j}, \beta_{j}\right)$ (which is necessarily horizontal) is tantamount to having a white marble $\circ$ at position $\alpha_{j}^{\mathrm{y}}=\beta_{j}^{\mathrm{y}}$ in 1 , see Figure 9(A). This can be achieved at the level of transfer matrices by multiplying the unconstrained transfer matrix $\Gamma_{a_{i}, b_{i}}\left(x_{i}\right)$ on the left by the projectors $\psi_{k}^{*} \psi_{k}$ with $k=\beta_{j}^{\mathrm{y}}, j=1 . . m$, and we get (46) upon anticommuting all $\psi^{*}$ 's to the left.

The case $m^{\prime} \neq 0$ is slightly more involved and requires the introduction of suitable "particle hopping" operators. Recall that the notation $\mathbf{m}^{(k)}$ denotes the Maya diagram obtained from $\mathbf{m}$ by inverting the color of the marble on site $k$. Let us consider an edge $e_{j}=\left(\gamma_{j}, \delta_{j}\right)$ : having this edge covered by a dimer implies that $\mathbf{l}_{\gamma_{j}^{\mathrm{y}}}=\mathbf{r}_{\delta_{j}^{\mathrm{y}}}=\bullet$, but the converse is not true. However there is a bijection between, on the one hand, admissible dimer configurations with boundary states $\mathbf{l}, \mathbf{r}$ containing $e_{j}$ and, on the other hand, admissible dimer configurations with boundary states $\mathbf{l}^{\left(\gamma_{j}^{\mathrm{y}}\right)}, \mathbf{r}^{\left(\delta_{j}^{\mathrm{y}}\right)}$, that necessarily contain the edge $e_{j}^{\prime}$ on the left of $\gamma_{j}$, see Figure 9 (BC). We deduce that, at the transfer matrix level, we can force the presence of $e_{j}$ by multiplying the unconstrained transfer matrix $\Gamma_{a_{i}, b_{i}}\left(x_{i}\right)$ by $\psi_{\gamma_{j}^{\mathrm{y}}}$ on the left and by $\psi_{\delta_{j}^{\mathrm{y}}}^{*}$ on the right (if $\mathbf{l}$ or $\mathbf{r}$ do not have black marbles at the required positions then $\langle\mathbf{l}|$ or $|\mathbf{r}\rangle$ will be killed by this $\psi$ or $\psi^{*}$, as it should be). More generally, the product $\left\langle\mathbf{l}\left|\psi_{\gamma_{1}^{y}} \cdots \psi_{\gamma_{m^{\prime}}^{\mathrm{y}}} \Gamma_{a_{i}, b_{i}}\left(x_{i}\right) \psi_{\delta_{m^{\prime}}^{\mathrm{y}}}^{*} \cdots \psi_{\delta_{1}^{\mathrm{y}}}^{*}\right| \mathbf{r}\right\rangle$ is nonzero if and only if there is a dimer configuration with boundary states $\mathbf{l}, \mathbf{r}$ containing all the $e_{j}, j=1 . . m^{\prime}$. In that case this configuration is unique and the product is equal to $x_{i}^{n}$, with $n$ the number of dimers on diagonal edges other than the $e_{j}$ (it is easily seen that the signs 


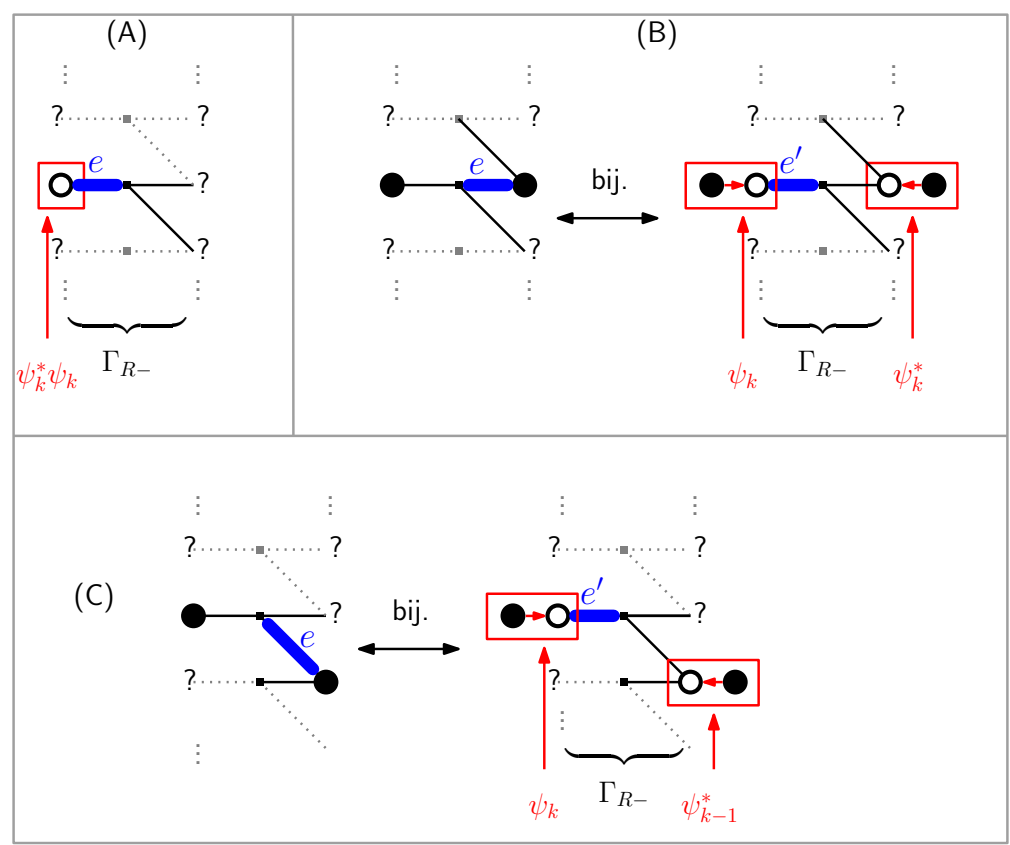

FiguRE 9. Localization of dimers in the case of the elementary graph or type $R-$ (the case of $R+$ is similar). Marbles (resp. edges) whose status is not fixed by the discussion are represented with question marks (resp. dotted lines). (A) There is a horizontal dimer in the left column if and only if the left odd vertex is occupied by a white marble. This marble is localized by applying the operator $\psi_{k}^{*} \psi_{k}$. (B) Configurations with a horizontal dimer in the right column are such that both marbles on this level are black (although this condition is not sufficient). They are in bijection with configurations with a dimer in the left column such that both marbles on this level are white. The operator $\psi_{k}^{*}\left(\right.$ resp. $\left.\psi_{k}\right)$ inserted on the right (resp. left) has the double effect of switching the color of marbles on the $k$-th level and of forcing the colors of these marbles. (C) The case of a diagonal dimer.

induced by the $\psi / \psi^{*}$ all cancel out). Upon reordering the $\psi^{*}$ 's in reverse order, multiplying by $x_{i}^{n_{i}}$ (to account for the weight of the dimers on diagonal edges in $\left.e_{j}, j=1 . . m^{\prime}\right)$ and multiplying by projectors on the left, we conclude that $(50)$ is true with $T_{i}$ given by 46 .

The case $a_{i}=L$ can be deduced from the discussion of the case $a_{i}=R$, by performing a central symmetry and exchanging the colors of the marbles. In other words, we simply need to take the dual of 46) (vertical symmetry) and conjugate with the involution $\omega$ acting on charged partitions/Maya diagrams (horizontal and color symmetry). There is a slight subtlety regarding the sign though, which can be treated using Proposition 10 . When taking the dual of $(46)$, the order of the operators is reversed, $\Gamma_{R, \pm}\left(x_{i}\right)$ is changed into $\Gamma_{R, \mp}\left(x_{i}\right)$ and each $\psi$ is changed into a $\psi^{*}$ (and vice versa). When conjugating by $\omega, \Gamma_{R, \mp}\left(x_{i}\right)$ is changed into $\Gamma_{L, \mp}\left(x_{i}\right)$ and each $\psi^{*}$ is changed back into a $\psi$ (and vice versa), up to a sign. By (34), the signs cancel out for horizontal edges in $E$, but combine into a sign -1 for each diagonal edge in $E$, which explains why the sign $(-1)^{k_{i}}$ is different in 47 . 
Remark 13. Similar bijective arguments are used in Appendix A to prove the commutation relations between bosonic and fermionic operators stated in Proposition 11

4.3. From the Schrödinger to the Heisenberg picture. Theorem 12 expresses $W(G ; \underline{x}, E)$ as a product of bosonic and fermionic operators taken between two vacuum states, which we may rewrite using a strategy coming from OR03, similar to that used in Section 3.3 for the proof of Theorem 1 move all $\Gamma_{+}$'s to the right, and all $\Gamma_{-}$'s to the left, so that they are absorbed by the vacuum states at the end. In this process, we first pick multiplicative factors due to the commutations between $\Gamma_{+}$'s and $\Gamma_{-}$'s: those are precisely the same as for the partition function $Z(G ; \underline{x})$. Second, the fermionic operators get "conjugated" by the $\Gamma$ 's crossing them. All this allows to rewrite

$$
W(G ; \underline{x}, E)=Z(G ; \underline{x})\left\langle\emptyset\left|\tilde{T}_{\ell} \tilde{T}_{\ell+1} \cdots \tilde{T}_{r}\right| \emptyset\right\rangle
$$

where, for all $i \in[\ell . . r]$, we set

$$
\tilde{T}_{i}=(-1)^{k_{i}} x_{i}^{n_{i}}\left(\prod_{j=1}^{m_{i}} \Psi^{*}\left(\beta_{i, j}\right)\right)\left(\prod_{j=1}^{m_{i}} \Psi\left(\alpha_{i, j}\right)\right)\left(\prod_{j=1}^{m_{i}^{\prime}} \Psi\left(\gamma_{i, j}\right)\right)\left(\prod_{j=1}^{m_{i}^{\prime}} \Psi^{*}\left(\delta_{i, j}\right)\right)
$$

with, for $\beta, \alpha$ respectively odd and even vertices of $G$,

$$
\left.\begin{array}{c}
\Psi^{*}(\beta)=\operatorname{Ad}\left(\prod_{\substack{i \leq\left\lfloor\beta^{\mathrm{x}} / 2\right\rfloor \\
b_{i}=+}} \Gamma_{a_{i},+}\left(x_{i}\right) \prod_{\substack{i \geq\left\lceil\beta^{\mathrm{x}} / 2\right\rceil \\
b_{i}=-}} \Gamma_{a_{i},-}^{-1}\left(x_{i}\right)\right) \psi_{\beta^{\mathrm{y}}}^{*}, \\
\Psi(\alpha)=\operatorname{Ad}\left(\prod_{\substack{i \leq \alpha^{\mathrm{x}} / 2 \text { if } a_{i}=L \\
i<\alpha^{\mathrm{x}} / 2 \text { if } a_{i}=R \\
b_{i}=+}} \Gamma_{a_{i},+}\left(x_{i}\right) \prod_{\substack{i \geq \alpha^{\mathrm{x}} / 2 \\
i>\alpha^{\mathrm{x}} / 2 \text { if } a_{i}=R \\
b_{i}=-}} \Gamma_{a_{i},-}^{-1}\left(x_{i}\right)\right.
\end{array}\right) \psi_{\alpha^{\mathrm{y}}} .
$$

Here Ad denotes the adjoint action:

$$
\operatorname{Ad}(A) B=A B A^{-1}
$$

with $A, B$ operators acting on $\mathcal{F}$, with $A$ invertible (recall that, by 17 ), this is the case for the $\Gamma$ 's). Two remarks are in order. First, we may put the $\Gamma_{+}$'s and the $\Gamma_{-}$'s in any order we want in $(53)$, as this does not change their adjoint action. Second, by Proposition 11, $\Psi^{*}(\beta)$ and $\Psi(\alpha)$ are (formal) linear combinations of $\psi^{*}$ 's and $\psi$ 's, respectively.

Remark 14. In physical terms, passing from the $\psi^{*} / \psi$ 's to the $\Psi^{*} / \Psi^{\prime}$ s can indeed be interpreted as going from the Schrödinger to the Heisenberg picture of quantum mechanics (the abscissa playing the role of time). It appears from $(53$ that creation and annihilation operators are naturally attached to respectively even and odd sites. An analogous situation appears in the path integral formalism: if $K$ denotes the Kasteleyn matrix of a finite planar bipartite graph, then the determinant of $K$, yielding the dimer partition function, can be written as a Grassmann-Berezin integral [D89]

$$
\operatorname{det} K=\int e^{\sum_{i, j} \xi_{i} K_{i, j} \xi_{j}^{*}} \prod_{i} d \xi_{i} \prod_{j} d \xi_{j}^{*}
$$

where the $\xi_{i}$ 's and $\xi_{j}^{*}$ are Grassmann variables attached to the even and odd vertices of the graph, respectively. Furthermore, the contribution of dimer configurations 
containing a given collection of edges $\left(i_{1}, j_{1}\right), \ldots,\left(i_{s}, j_{s}\right)$ is proportional to

$$
\int \xi_{i_{1}} \xi_{j_{1}}^{*} \cdots \xi_{i_{s}} \xi_{j_{s}}^{*} e^{\sum_{i, j} \xi_{i} K_{i, j} \xi_{j}^{*}} \prod_{i} d \xi_{i} \prod_{j} d \xi_{j}^{*} .
$$

In other words, dimer correlations are given by the expectation value of a product of fermionic operators, whose form is reminiscent of (52). Note however that the approach followed in this paper is more akin to canonical quantization.

4.4. Proof of Theorem 5. By (51) and (52), the ratio

$$
P_{G ; \underline{x}}(E)=\frac{W(G ; \underline{x}, E)}{Z(G ; \underline{x})}
$$

is given by a product of fermionic operators taken between two vacuum states. This can be rewritten as a determinant using Wick's formula, as follows.

For an even vertex $\alpha$ and an odd vertex $\beta$, define the naturally ordered product (or "time-ordered product") of $\Psi(\alpha)$ and $\Psi^{*}(\beta)$ by

$$
\mathcal{T}\left(\Psi(\alpha), \Psi^{*}(\beta)\right)= \begin{cases}\Psi(\alpha) \Psi^{*}(\beta) & \text { if } \alpha^{\mathrm{x}}<\beta^{\mathrm{x}} \\ -\Psi^{*}(\beta) \Psi(\alpha) & \text { if } \alpha^{\mathrm{x}}>\beta^{\mathrm{x}}\end{cases}
$$

We may more generally consider the naturally ordered product of more than two $\Psi$ or $\Psi^{*}$, by ordering them according to the abscissa of their argument and multiplying by the sign of the corresponding permutation (note that operators with the same abscissa anticommute, hence their order is irrelevant). Observe that $\tilde{T}_{\ell} \tilde{T}_{\ell+1} \cdots \tilde{T}_{r}$ is, up to a factor, the naturally ordered product of the $\Psi$ and $\Psi^{*}$ associated with the endpoints of the edges of $E$. Denoting now by $\left\{e_{1}, \ldots, e_{s}\right\}$ the edges of $E$, with $e_{i}=\left(\alpha_{i}, \beta_{i}\right)$, and by $H(E)$ the number of horizontal edges in $E$ whose right endpoint is at an even abscissa, we have

$$
\begin{aligned}
& P_{G ; \underline{x}}(E)=(-1)^{H(E)}\left(\prod_{i=\ell}^{r} x_{i}^{n_{i}}\right)\left\langle\emptyset\left|\mathcal{T}\left(\Psi\left(\alpha_{1}\right), \Psi^{*}\left(\beta_{1}\right), \ldots, \Psi\left(\alpha_{s}\right), \Psi^{*}\left(\beta_{s}\right)\right)\right| \emptyset\right\rangle \\
& =(-1)^{H(E)}\left(\prod_{i=\ell}^{r} x_{i}^{n_{i}}\right) \operatorname{det}_{1 \leq i, j \leq s}\left\langle\emptyset\left|\mathcal{T}\left(\Psi\left(\alpha_{i}\right), \Psi^{*}\left(\beta_{j}\right)\right)\right| \emptyset\right\rangle
\end{aligned}
$$

where we apply Wick's formula to pass from the first to the second line, and where the sign $(-1)^{H(E)}$ arises from the reordering of the fermionic operators (in particular, dimers having their right endpoint at an even abscissa appear in the "wrong order" in the naturally ordered product, but the resulting sign is cancelled in the case of diagonal dimers by that present in Theorem 12. For completeness, we provide a detailed derivation of Wick's formula in Appendix B To complete the proof of Theorem 5, it remains to check that

$$
\mathcal{C}_{\alpha, \beta}=\left\langle\emptyset\left|\mathcal{T}\left(\Psi(\alpha), \Psi^{*}(\beta)\right)\right| \emptyset\right\rangle
$$

has the announced expression 10 . At this stage we need to discuss a bit analyticity conditions (so far all our computations were done by treating the weights $x_{i}$ 's as formal variables). Recall that we aim at proving Theorem 5 under the mere assumption that the partition function $Z(G ; \underline{x})$ is a convergent sum, which boils down to the conditions (6) and (7) (see again Remark 6).

Let us temporarily strengthen (6) into the condition

$$
x_{i} x_{j}<1 \text { for all } i<j \text { such that } b_{i}=+ \text { and } b_{j}=-
$$


(that is, we also impose $x_{i} x_{j}<1$ when $a_{i} \neq a_{j}$ ). We introduce the quantities

$$
\rho_{+}(k)=\inf _{\substack{i: b_{i}=+2 i \leq k}}\left(\frac{1}{x_{i}}\right), \quad \rho_{-}(k)=\sup _{\substack{j: b_{j}=-2 j \geq k}} x_{j},
$$

which are nonnegative nonincreasing functions of $k$, such that $\rho_{-}(k)<\rho_{+}(k)$ by (61) and (7). Recalling the definition (31) of the fermionic generating functions $\psi(z)$ and $\psi^{*}(z)$, the definition (53) of $\Psi(\alpha)$ and $\Psi^{*}(\beta)$, and the bosonic-fermionic commutation relations of Proposition 11, we may write

$$
\Psi(\alpha)=\frac{1}{2 i \pi} \oint_{C_{z}} \frac{F_{\alpha^{\mathrm{x}}}(z) \psi(z)}{z^{\alpha^{\mathrm{y}}}} \frac{d z}{z}, \quad \Psi^{*}(\beta)=\frac{1}{2 i \pi} \oint_{C_{w}} \frac{\psi^{*}(w) w^{\beta^{\mathrm{y}}}}{F_{\beta^{\mathrm{x}}}(w)} \frac{d w}{w}
$$

with $C_{z}$ (resp. $\left.C_{w}\right)$ a circle centered at 0 with radius comprised between $\rho_{-}\left(\alpha^{\mathrm{x}}\right)$ and $\rho_{+}\left(\alpha^{\mathrm{x}}\right)$ (resp. between $\rho_{-}\left(\beta^{\mathrm{x}}\right)$ and $\left.\rho_{+}\left(\beta^{\mathrm{x}}\right)\right)$. We deduce that $\left\langle\emptyset\left|\mathcal{T}\left(\Psi(\alpha), \Psi^{*}(\beta)\right)\right| \emptyset\right\rangle$ is equal to the wanted expression 10 provided that

$$
\left\langle\emptyset\left|\mathcal{T}\left(\psi(z), \psi^{*}(w)\right)\right| \emptyset\right\rangle=\frac{\sqrt{z w}}{z-w} .
$$

But this readily follows from the definition $(58)$ of the naturally ordered product and from the relations $(33)$, provided that we take the radius of $C_{z}$ to be strictly larger than that of $C_{w}$ if $\alpha^{\mathrm{x}}<\beta^{\mathrm{x}}$, and vice versa otherwise. We may now freely deform the contours $C_{z}$ and $C_{w}$, as long as we hit no pole of the integrand: this establishes (10) under the conditions (i)-(iii) for the contours, hence Theorem 5 under the assumption 61 .

We now explain how to relax this assumption into (6). We proceed by multiplying each $x_{i}$ by a factor $t \in[0,1]$, and noting that the assumption (61) hence the identity (11) are satisfied for $t$ small enough. We will show that both sides of (11) have an analytic continuation in $t$ to a domain containing the closed unit disk. As apparent from Theorem 1 , this is the case for the partition function $Z(G ; t \underline{x})$ (provided that (6) is satisfied, of course). The quantity $W(G ; t \underline{x}, E)$, being a sum over a restricted subset of configurations, is analytic too, hence so does $P_{G ; t \underline{x}}(E)$ which is the lefthand side of (11). As for the right-hand side, let us show that we may find two contours $C_{z}$ and $C_{w}$ such that $\mathcal{C}_{\alpha, \beta}^{(t)}$, as defined by 10 with the $x_{i}$ 's multiplied by $t$, is manifestly analytic as $t$ varies over the unit disk. For $t=1$, let us introduce the quantities

$$
\begin{array}{ll}
\rho_{R+}(k)=\inf _{\substack{i:\left(a_{i}, b_{i}\right)=(R,+) \\
2 i<k}}\left(\frac{1}{x_{i}}\right), & \rho_{L-}(k)=\sup _{j:\left(a_{j}, b_{j}\right)=(L,-)} x_{j}>k \\
\rho_{L+}(k)=\inf _{\substack{i:\left(a_{i}, b_{i}\right)=(L,+) \\
2 i \leq k}}\left(\frac{1}{x_{i}}\right), & \rho_{R-}(k)=\sup _{\substack{j:\left(a_{j}, b_{j}\right)=(R,-) \\
2 j \geq k}} x_{j},
\end{array}
$$

which are refinements of $\rho_{+}(k)$ and $\rho_{-}(k)$. We take $C_{z}$ to be a circle centered on the real axis, surrounding the real interval $\left[-\rho_{R-}\left(\alpha^{\mathrm{x}}\right), 0\right]$ but not intersecting $\left[\rho_{L+}\left(\alpha^{\mathrm{x}}\right),+\infty\left[\right.\right.$. Similarly, we take $C_{w}$ to be a circle centered on the real axis, surrounding the real interval $\left[0, \rho_{L-}\left(\beta^{\mathrm{x}}\right)\right]$ but not intersecting $\left.]-\infty,-\rho_{R+}\left(\beta^{\mathrm{x}}\right)\right]$. We furthermore want $C_{z}$ and $C_{w}$ not to intersect, with $C_{z}$ surrounding $C_{w}$ iff $\alpha^{x}<\beta^{x}$ : this is possible because the functions $\rho_{L \pm}$ are decreasing with $k$ and such that $\rho_{L-}(k)<\rho_{L+}(k)$ for all $k$ hence, for $\alpha^{x}<\beta^{x}$, we have $\rho_{L-}\left(\beta^{x}\right) \leq$ $\rho_{L-}\left(\alpha^{x}\right)<\rho_{L+}\left(\alpha^{x}\right)$ so that the "window" $] \rho_{L-}\left(\beta^{x}\right), \rho_{L+}\left(\alpha^{x}\right)\left[\right.$ in which both $C_{z}$ and $C_{w}$ must pass is nonempty, see Figure 10 (and the case $\alpha^{x}>\beta^{x}$ is treated by a similar reasoning on $\left.\rho_{R \pm}\right)$. Observe that these contours satisfy precisely the conditions (i)-(iii) stated below equation (10). Keeping these contours fixed, we let $t$ vary in the interval $[0,1]$ : no pole of $G_{\alpha^{\mathrm{x}}, \beta^{\mathrm{x}}}^{(t)}(z, w)$ ever hits the contours, the 


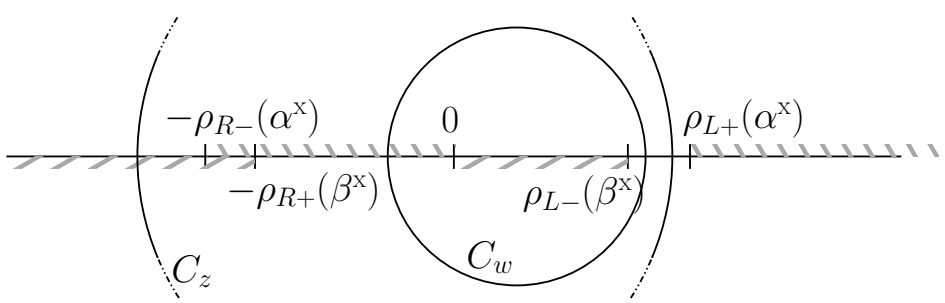

Figure 10. The contours $C_{z}$ and $C_{w}$ in the case $\beta^{\mathrm{x}}>\alpha^{\mathrm{x}}$. The upper (resp. lower) dashed regions are the intervals that the contour $C_{z}$ (resp. $C_{w}$ ) must avoid. On this figure it is assumed that $\rho_{R-}\left(\alpha^{\mathrm{x}}\right)>\rho_{R+}\left(\beta^{\mathrm{x}}\right)$ but the converse can also hold.

conditions (i)-(iii) remain satisfied, and we conclude that 10 defines the wanted analytic continuation of $\mathcal{C}_{\alpha, \beta}^{(t)}$.

4.5. Inverse Kasteleyn matrix. Let us now connect Theorem 5 to the general Kasteleyn theory for the dimer model on planar bipartite graphs. We mention that a similar connection was already observed in [OR07] for the case of lozenge tilings corresponding to skew plane partitions, see also [BF14, Section 5].

Definition 15. Let $G=(V, E)$ be a planar bipartite graph with no multiple edges. Consider a collection of weights on the edges $\omega: E \rightarrow \mathbb{R}_{+}$.

A Kasteleyn orientation of $G$ is a map $\eta: E \rightarrow\{-1,1\}$ such that for any face $F$ the product over the edges surrounding $F$ gives :

$$
\prod_{e \in F} \eta(e)=\left\{\begin{array}{lll}
1 & \text { if } F \text { is of degree } 2 & (\bmod 4) \\
-1 & \text { if } F \text { is of degree } 0 & (\bmod 4)
\end{array}\right.
$$

The Kasteleyn matrix of $G$ associated to $\eta$ is the matrix $\mathcal{K}$ whose rows (resp. columns) are indexed by white (resp. black) vertices of $G$, such that for any couple $(w, b)$ of a white vertex and a black vertex,

$$
\mathcal{K}(w, b)=\left\{\begin{array}{ll}
0 & \text { if } w \text { not adjacent to } b \\
\eta(w, b) \omega(w, b) & \text { if there is an edge }(w, b)
\end{array} .\right.
$$

Local statistics for dimers are known to be given by determinants of submatrices of the Kasteleyn matrix [Kas61, Per69, Ken97]:

Theorem 16. Let $(G, \omega)$ be a finite weighted planar bipartite graph. The probability that the dimers $e_{1}=\left(w_{1}, b_{1}\right), \ldots, e_{s}=\left(w_{s}, b_{s}\right)$ are present in a random dimer configuration sampled with a probability proportional to its weight is:

$$
P\left(e_{1}, \ldots, e_{s}\right)=\left(\prod_{i=1}^{s} \mathcal{K}\left(w_{i}, b_{i}\right)\right) \operatorname{det}\left(\mathcal{K}^{-1}\left(b_{i}, w_{j}\right)\right)_{1 \leq i, j \leq s} .
$$

Let us go back to the rail yard graphs. In this case, the black (resp. white) vertices are the even (resp. odd) vertices. Recall that in pure dimer coverings of a rail yard graph $G$, all the odd vertices with negative (resp. positive) ordinate on the left (resp. right) boundary are unmatched. These pure dimer coverings on $G$ correspond to classical dimer coverings with finitely many diagonal edges on the graph $\tilde{G}$, where those unmatched vertices and the edges attached to them are removed. Compare Figure 11 and Figure 4. 


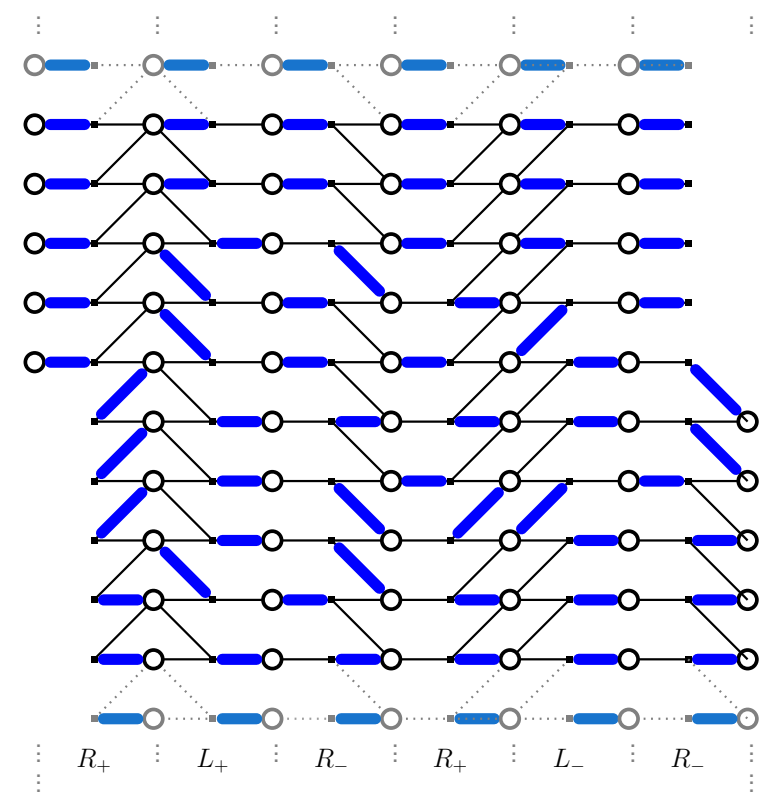

FIGURE 11. The perfect matching of the modified rail graph corresponding to the pure dimer covering of Figure 4.

Let us denote by $\mathfrak{M}$ the set of matched odd vertices of $G$. Define $\eta: E \rightarrow\{-1,1\}$ by

$$
\eta(e)= \begin{cases}-1 & \text { if } e \text { is a horizontal edge whose right end is an even vertex } \\ 1 & \text { otherwise }\end{cases}
$$

It is easy to check that $\eta$ is a Kasteleyn orientation on $\tilde{G}$ (here the faces have degree 4,6 or 8). Construct the infinite Kasteleyn matrix $\mathcal{K}$ on that graph as in (67). The restriction $\tilde{\mathcal{K}}$ of $\mathcal{K}$ to rows indexed by $\mathfrak{M}$ is a Kasteleyn matrix for $\tilde{G}$. We now relate our correlation kernel $\mathcal{C}$ to the infinite Kasteleyn matrix $\mathcal{K}$ :

Theorem 17. Let $G=\operatorname{RYG}(\ell, r, \underline{a}, \underline{b})$ be a rail yard graph and $\mathcal{K}$ be its Kasteleyn matrix for the previously defined orientation. Then if $\mathcal{C}$ is the matrix defined in Theorem 5, we have:

(1) for any $\alpha, \alpha^{\prime}$ even vertices, $(\mathcal{C K})_{\alpha, \alpha^{\prime}}=\delta_{\alpha, \alpha^{\prime}}$;

(2) for any $\beta, \beta^{\prime}$ odd vertices in $\mathfrak{M},(\mathcal{K C})_{\beta, \beta^{\prime}}=\delta_{\beta, \beta^{\prime}}$.

We state a lemma that will be useful to prove the theorem. Recall that we have a bosonic operator $\Gamma_{R \pm}\left(x_{m}\right)\left(\right.$ resp. $\left.\Gamma_{L \pm}\left(x_{m}\right)\right)$ at position $(2 m+1 / 2,0)$ (resp. $(2 m-1 / 2,0))$ when $a_{m}=R$ (resp. $\left.a_{m}=L\right)$. To simplify notations, we will also place a bosonic operator $I d$ at every position $(2 m-1 / 2,0)($ resp. $(2 m+1 / 2,0))$ when $a_{m}=R$ (resp. $a_{m}=L$ ). This does not change the naturally ordered product of operators, and now we have one bosonic operator at each half-integer abscissa in $[2 \ell-1, \ldots, 2 r+1]$. Let $B^{-}\left(\right.$resp. $\left.B^{+}\right)$be the bosonic operator at abscissa $i-\frac{1}{2}$ (resp. $i+\frac{1}{2}$ ). Denote by $x \sim y$ the fact that two vertices $x$ and $y$ are adjacent in G.

Lemma 18. We have the following properties: 
(1) Let $\alpha$ be an even vertex at position $(i, k)$. Then

$$
\begin{aligned}
& \sum_{\substack{\beta \sim \alpha \\
\beta^{\mathrm{x}}<i}} \mathcal{K}(\beta, \alpha) \psi_{\beta^{\mathrm{y}}}^{*} B^{-}=-B^{-} \psi_{k}^{*} ; \\
& \sum_{\substack{\beta \sim \alpha \\
\beta^{\mathrm{x}}>i}} \mathcal{K}(\beta, \alpha) B^{+} \psi_{\beta^{\mathrm{y}}}^{*}=\psi_{k}^{*} B^{+} .
\end{aligned}
$$

(2) Let $\beta$ be an odd vertex at position $(i, k)$. Then

$$
\begin{aligned}
& \sum_{\substack{\alpha \sim \beta \\
\alpha^{x}<i}} \mathcal{K}(\beta, \alpha) \psi_{\alpha^{y}} B^{-}=B^{-} \psi_{k} ; \\
& \sum_{\substack{\alpha \sim \beta \\
\alpha^{x}>i}} \mathcal{K}(\beta, \alpha) B^{+} \psi_{\alpha^{y}}=-\psi_{k} B^{+} .
\end{aligned}
$$

Proof. We will prove only identity $(70)$. The other identities can be proved in a similar way. We distinguish three cases:

Case 1: $B^{-}=I d$. To its left, $\alpha$ has only one neighbour $\beta$, which is at height $k$. We conclude from the fact that $\mathcal{K}(\beta, \alpha)=-1$.

Case 2: $B^{-}=\Gamma_{L-}(x)$. To its left, $\alpha$ has one neighbour $\beta_{1}$ at height $k$ and one neighbour $\beta_{2}$ at height $k-1$. We compute

$$
\begin{aligned}
\sum_{\substack{\beta \sim \alpha \\
\beta^{\times}<i}} \mathcal{K}(\beta, \alpha) \psi_{\beta}^{*} \Gamma_{L-}(x) & =\mathcal{K}\left(\beta_{1}, \alpha\right) \psi_{k}^{*} \Gamma_{L-}(x)+\mathcal{K}\left(\beta_{2}, \alpha\right) \psi_{k-1}^{*} \Gamma_{L-}(x) \\
& =-\psi_{k}^{*} \Gamma_{L-}(x)+x \psi_{k-1}^{*} \Gamma_{L-}(x) \\
& =-\left[z^{-k}\right] \psi^{*}(z) \Gamma_{L-}(x)+\left[z^{-k+1}\right] x \psi^{*}(z) \Gamma_{L-}(x) \\
& =-\left[z^{-k}\right] \psi^{*}(z) \Gamma_{L-}(x)+\left[z^{-k}\right] \frac{x}{z} \psi^{*}(z) \Gamma_{L-}(x) \\
& =-\left[z^{-k}\right]\left(1-\frac{x}{z}\right) \psi^{*}(z) \Gamma_{L-}(x) \\
& =-\left[z^{-k}\right] \Gamma_{L-}(x) \psi^{*}(z) \\
& =-\Gamma_{L-}(x) \psi_{k}^{*},
\end{aligned}
$$

where we used proposition 11 to switch $\psi^{*}(z)$ and $\Gamma_{L-}(x)$.

Case 3: $B^{-}=\Gamma_{L+}(x)$. In the proof of case 2 , replace $\Gamma_{L-}(x)$ by $\Gamma_{L+}(x), k-1$ by $k+1$ and $\frac{x}{z}$ by $x z$.

We can now prove Theorem 17

Proof of Theorem 17 . In this proof, to make notations lighter, $Z(G ; \underline{x})$ will be abbreviated as $Z$. Let us prove the first part of the theorem. Fix two even vertices $\alpha$ at position $(i, k)$ and $\alpha^{\prime}$ at position $\left(i^{\prime}, k^{\prime}\right)$. Then :

$$
(\mathcal{C K})\left(\alpha^{\prime}, \alpha\right)=\sum_{\beta \sim \alpha} \mathcal{C}\left(\alpha^{\prime}, \beta\right) \mathcal{K}(\beta, \alpha) .
$$

This sum has at most three nonzero terms, corresponding to the three odd neighbours of $\alpha$, at the abscissas $i-1$ and $i+1$. We now distinguish according to the 
position of $i^{\prime}$ relatively to $i$.

Case 1: $i^{\prime}<i$.

If $\beta \sim \alpha$ is at abscissa $i-1, \mathcal{C}\left(\alpha^{\prime}, \beta\right)$ can be written in the following form :

$$
\mathcal{C}\left(\alpha^{\prime}, \beta\right)=\frac{1}{Z}\left\langle\emptyset\left|\Gamma^{(1)} \psi_{k^{\prime}} \Gamma^{(2)} \psi_{\beta^{\mathrm{y}}}^{*} B^{-} B^{+} \Gamma^{(3)}\right| \emptyset\right\rangle,
$$

where $\Gamma^{(1)}, \Gamma^{(2)}$ and $\Gamma^{(3)}$ are the products of the bosonic operators located respectively before the abscissa $i^{\prime}$, between the abscissas $i^{\prime}$ and $i-1$ and after the abscissa $i+1$. form:

Similarly, if $\beta \sim \alpha$ is at abscissa $i+1, \mathcal{C}\left(\alpha^{\prime}, \beta\right)$ can be written in the following

$$
\mathcal{C}\left(\alpha^{\prime}, \beta\right)=\frac{1}{Z}\left\langle\emptyset\left|\Gamma^{(1)} \psi_{k^{\prime}} \Gamma^{(2)} B^{-} B^{+} \psi_{\beta^{y}}^{*} \Gamma^{(3)}\right| \emptyset\right\rangle .
$$

For each $\beta \sim \alpha$ appearing in the sum of equation $(74)$, we are going to move the $\psi_{\beta^{\mathrm{y}}}^{*}$ between $B^{-}$and $B^{+}$. We separate the cases $\beta^{\mathrm{x}}<i$ and $\beta^{\mathrm{x}}>i$ to apply part 1 of the lemma:

$$
\begin{aligned}
(\mathcal{C K})\left(\alpha^{\prime}, \alpha\right) & =\sum_{\beta \sim \alpha} \mathcal{K}(\beta, \alpha) \mathcal{C}\left(\alpha^{\prime}, \beta\right) \\
& =\sum_{\substack{\beta \sim \alpha \\
\beta^{\mathrm{x}}<i}} \mathcal{K}(\beta, \alpha) \mathcal{C}\left(\alpha^{\prime}, \beta\right)+\sum_{\substack{\beta \sim \alpha \\
\beta^{\mathrm{x}}>i}} \mathcal{K}(\beta, \alpha) \mathcal{C}\left(\alpha^{\prime}, \beta\right) \\
& =-\frac{1}{Z}\left\langle\emptyset\left|\Gamma^{(1)} \psi_{k^{\prime}} \Gamma^{(2)} B^{-} \psi_{k}^{*} B^{+} \Gamma^{(3)}\right| \emptyset\right\rangle+\frac{1}{Z}\left\langle\emptyset\left|\Gamma^{(1)} \psi_{k^{\prime}} \Gamma^{(2)} B^{-} \psi_{k}^{*} B^{+} \Gamma^{(3)}\right| \emptyset\right\rangle \\
& =0 .
\end{aligned}
$$

Case 2: $i^{\prime}>i$.

Here $\psi_{k^{\prime}}$ is to the left of any $\psi_{\beta^{y}}^{*}$, so each $\mathcal{C}\left(\alpha^{\prime}, \beta\right)$ comes with a minus sign. Using here again part 1 of the lemma to move $\psi_{\beta^{y}}^{*}$ between $B^{-}$and $B^{+}$for all three terms, we get similarly that $(\mathcal{C K})\left(\alpha^{\prime}, \alpha\right)=0$ in this case.

Case 3: $i^{\prime}=i$.

If $\beta$ is at abscissa $i-1$, then $\mathcal{C}\left(\alpha^{\prime}, \beta\right)$ can be written in the following form:

$$
\mathcal{C}\left(\alpha^{\prime}, \beta\right)=-\frac{1}{Z}\left\langle\emptyset\left|\Gamma^{(1)} \psi_{\beta^{\mathrm{y}}}^{*} B^{-} \psi_{k^{\prime}} B^{+} \Gamma^{(2)}\right| \emptyset\right\rangle,
$$

where the minus sign comes from the fact that $\psi_{\beta^{y}}^{*}$ appears before $\psi_{k^{\prime}}$.

If $\beta \sim \alpha$ is at abscissa $i+1$, we have:

$$
\mathcal{C}\left(\alpha^{\prime}, \beta\right)=\frac{1}{Z}\left\langle\emptyset\left|\Gamma^{(1)} B^{-} \psi_{k^{\prime}} B^{+} \psi_{\beta^{y}}^{*} \Gamma^{(2)}\right| \emptyset\right\rangle .
$$

Applying again part 1 of the lemma, we get:

$$
\begin{aligned}
(\mathcal{C K})\left(\alpha^{\prime}, \alpha\right) & =\sum_{\beta \sim \alpha} \mathcal{K}(\beta, \alpha) \mathcal{C}\left(\alpha^{\prime}, \beta\right) \\
& =\sum_{\substack{\beta \sim \alpha \\
\beta^{\mathrm{x}}<i}} \mathcal{K}(\beta, \alpha) \mathcal{C}\left(\alpha^{\prime}, \beta\right)+\sum_{\substack{\beta \sim \alpha \\
\beta^{\mathrm{x}}>i}} \mathcal{K}(\beta, \alpha) \mathcal{C}\left(\alpha^{\prime}, \beta\right) \\
& =\frac{1}{Z}\left\langle\emptyset\left|\Gamma^{(1)} \Gamma^{(2)} B^{-} \psi_{k}^{*} \psi_{k^{\prime}} B^{+} \Gamma^{(3)}\right| \emptyset\right\rangle+\frac{1}{Z}\left\langle\emptyset\left|\Gamma^{(1)} \Gamma^{(2)} B^{-} \psi_{k^{\prime}} \psi_{k}^{*} B^{+} \Gamma^{(3)}\right| \emptyset\right\rangle .
\end{aligned}
$$

Using that $\psi_{k}^{*} \psi_{k^{\prime}}+\psi_{k^{\prime}} \psi_{k}^{*}=\delta_{k, k^{\prime}}$ (Proposition9) and that

$$
Z=\left\langle\emptyset\left|\Gamma^{(1)} \Gamma^{(2)} B^{-} B^{+} \Gamma^{(3)}\right| \emptyset\right\rangle,
$$


We conclude that in this third case, $(\mathcal{C K})\left(\alpha^{\prime}, \alpha\right)=\delta_{k, k^{\prime}}$. This concludes the proof of part 1 of the theorem : we have shown that for any even vertices $\alpha$ and $\alpha^{\prime},(\mathcal{C K})\left(\alpha^{\prime}, \alpha\right)=\delta_{\alpha^{\prime}, \alpha}$.

To prove part 2 of the theorem, note that if $\beta$ and $\beta^{\prime}$ are two odd vertices in $\mathfrak{M}$, then

$$
(\mathcal{K C})\left(\beta, \beta^{\prime}\right)=\sum_{\alpha \sim \beta} \mathcal{K}(\beta, \alpha) \mathcal{C}\left(\alpha, \beta^{\prime}\right) .
$$

We will only treat the case when $\beta$ is on the left boundary. The case when $\beta$ is on the right boundary is similar to this one. The case when $\beta$ is in the bulk is similar to the proof of part 1 of the theorem, by making use this time of part 2 of the lemma. Let $(2 \ell-1, k)$ be the coordinates of $\beta$ and $\left(i^{\prime}, k^{\prime}\right)$ be the coordinates of $\beta^{\prime}$. Since $\beta \in \mathfrak{M}$, we have $k>0$. Note that $\beta$ has neighbours only to its right. Again we distinguish according to the position of $\beta^{\prime}$.

Case $1: i^{\prime}>2 \ell-1$.

If $\alpha \sim \beta, \mathcal{C}\left(\alpha, \beta^{\prime}\right)$ can be written in the following form :

$$
\mathcal{C}\left(\alpha, \beta^{\prime}\right)=\frac{1}{Z}\left\langle\emptyset\left|B^{+} \psi_{\alpha^{y}} \Gamma^{(1)} \psi_{k^{\prime}}^{*} \Gamma^{(2)}\right| \emptyset\right\rangle
$$

Using the second equation of part 2 of the lemma, we obtain:

$$
\begin{aligned}
(\mathcal{K C})\left(\beta, \beta^{\prime}\right) & =\sum_{\alpha \sim \beta} \mathcal{K}(\beta, \alpha) \mathcal{C}\left(\alpha, \beta^{\prime}\right) \\
& =\sum_{\substack{\alpha \sim \beta \\
\alpha^{\alpha}>2 \ell-1}} \mathcal{K}(\beta, \alpha) \mathcal{C}\left(\alpha, \beta^{\prime}\right) \\
& =-\frac{1}{Z}\left\langle\emptyset\left|\psi_{k} B^{+} \Gamma^{(1)} \psi_{k^{\prime}}^{*} \Gamma^{(2)}\right| \emptyset\right\rangle .
\end{aligned}
$$

Since $k>0$, we have $\langle\emptyset| \psi_{k}=0$. So in this case, $(\mathcal{K C})\left(\beta, \beta^{\prime}\right)=0$.

Case 2 : $i^{\prime}=2 \ell-1$.

If $\alpha \sim \beta, \mathcal{C}\left(\alpha, \beta^{\prime}\right)$ can be written in the following form :

$$
\mathcal{C}\left(\alpha, \beta^{\prime}\right)=-\frac{1}{Z}\left\langle\emptyset\left|\psi_{k^{\prime}}^{*} B^{+} \psi_{\alpha^{y}} \Gamma^{(1)}\right| \emptyset\right\rangle .
$$

Using the second equation of part 2 of the lemma, we obtain :

$$
(\mathcal{K C})\left(\beta, \beta^{\prime}\right)=+\frac{1}{Z}\left\langle\emptyset\left|\psi_{k^{\prime}}^{*} \psi_{k} B^{+} \Gamma^{(1)}\right| \emptyset\right\rangle .
$$

Using Proposition 9, we get :

$$
\begin{aligned}
(\mathcal{K C})\left(\beta, \beta^{\prime}\right) & =-\frac{1}{Z}\left\langle\emptyset\left|\psi_{k} \psi_{k^{\prime}}^{*} B^{+} \Gamma^{(1)}\right| \emptyset\right\rangle+\delta_{k, k^{\prime}} \frac{1}{Z}\left\langle\emptyset\left|B^{+} \Gamma^{(1)}\right| \emptyset\right\rangle \\
& =0+\delta_{k, k^{\prime}} \frac{1}{Z} Z=\delta_{k, k^{\prime}} .
\end{aligned}
$$

To sum up, if $\beta$ is an odd vertex in $\mathfrak{M}$ on the left boundary and $\beta^{\prime}$ is any other odd vertex in $\mathfrak{M},(\mathcal{K C})_{\left(\beta, \beta^{\prime}\right)}=\delta_{\beta, \beta^{\prime}}$.

Remark 19. If $\tilde{\mathcal{C}}$ (resp. $\tilde{\mathcal{K}}$ ) is the restriction of $\mathcal{C}$ (resp. $\mathcal{K}$ ) to columns (resp. rows) indexed by the set $\mathfrak{M}$ of matched odd vertices, then $\tilde{\mathcal{C}}$ is a left and right inverse of $\tilde{\mathcal{K}}$. Indeed, the extra terms coming from vertices not in $\mathfrak{M}$ vanish when evaluated against left and right vacuums. 


\section{LOZENGE AND DOMINO TILINGS}

In this section we discuss how the RYG dimer model gives rise, upon taking a constant or an alternating $L R$-sequence and performing a simple change of coordinates, to a class of lozenge or domino tilings of the plane, respectively. The class of lozenge tilings, discussed in Section 5.1. contains in particular plane partitions and was already studied in OR03, OR07. The class of domino tilings, discussed in Section 5.2, is that of steep tilings [BCC14], and contains tilings of the Aztec diamond and pyramid partitions as special cases.

5.1. Lozenge tilings. In this section we consider the case where the $L R$-sequence $\underline{a}$ is constant. We assume that $\underline{a}=L^{r-\ell+1}$ (it is easy to check that taking $\underline{a}=R^{r-\ell+\overline{1}}$ gives rise to the same model up to a vertical reflection and an inversion of the sign sequence). In this case the only two elementary RYG involved are of type $L+$ and $L-$. According to the discussion of Section 3, the transfer matrix operators corresponding to these two graphs can be interpreted as the operators $\Gamma_{L+}$ and $\Gamma_{L-}$, whose action on the Bosonic Fock space interlaces a partition "upwards" or "downwards" respectively, see (14). It follows that, for each sign sequence $\underline{b} \in\{+,-\}^{r-\ell+1}$, admissible dimer coverings of $\mathrm{RYG}(\ell, r, \underline{a}, \underline{b})$ are in bijection with sequences $\left(\lambda^{(i)}\right)_{\ell<i<r+1}$ of integer partitions such that $\lambda^{(i)} \succsim \lambda^{(i+1)}$ where the interlacing relation is $\prec$ or $\succ$ if $b_{i}=+$ or $b_{i}=-$, respectively. The correspondence goes via Maya diagrams and is the one described in Section3. It is well-known that such sequences are in bijection with certain lozenge tilings of the plane, see e.g. OR03, so the reader may be in familiar ground. In the rest of Section 5.1, we will just sketch how to recover those lozenge tilings from the rail yard graphs.

We first apply the following coordinate transformation to each vertex of the rail yard graph $\operatorname{RYG}(\ell, r, \underline{a}, \underline{b})$ :

$$
(2 \ell-1+x, y) \longmapsto\left(2 \ell-1+\frac{1}{2}\lceil x / 2\rceil+\frac{\sqrt{5}}{4}\lfloor x / 2\rfloor, y-\frac{1}{2} \sum_{i=1}^{\lceil x / 2\rceil}(-1)^{b_{i}}\right) .
$$

Figure 13 displays the effect of this transformation on the elementary graphs of type $L+$ and $L-$. The transformation is designed in such a way that after the transformation all angles between incident edges are equal to $\frac{2 \pi}{3}$, and that all edges have equal length (equal to $\frac{\sqrt{5}}{4}$ ). Therefore the concatenation of these graphs generate a portion of the regular hexagonal lattice, see Figure 14-Left. Since the planar dual of the hexagonal lattice is a triangular lattice, any dimer covering of the hexagonal lattice induces a covering of this triangular lattice by lozenges (each dimer connects two vertices in the primal, that correspond to two triangles in the dual, and the union of these two triangles forms a lozenge). See Figure 14-Right. We thus recover the promised class of lozenge tilings of the plane.

Note that the fundamental covering of $\operatorname{RYG}(\ell, r, \underline{a}, \underline{b})$ projects (via the coordinate transformation (84) and the dualization to lozenges) to a lozenge tiling in which all lozenges under a certain separating path are of "horizontal" type, while all lozenges above this path are of one of the two "vertical" types, see Figure 15. This separating path is the image under the transformation (84) of the horizontal axis in the original embedding of $\operatorname{RYG}(\ell, r, \underline{a}, \underline{b})$. Equivalently, this path is a lattice path taking up $(+)$ or down (-) steps, encoded by the sequence $\underline{b}$. This path and its image are represented by red dotted paths on the figures of this section.

Finally, note that Figure 15 can naturally be seen as a 3-dimensional picture, namely as a portion of the boundary of the region $\left(\mathbb{R}_{+}^{2} \backslash \lambda\right) \times \mathbb{R}_{+}$, where $\lambda$ is the shape of an integer partition. Here the contour of the shape $\lambda$ coincides with the separating path just mentioned, i.e. the partition $\lambda$ is encoded in Russian notation by the 


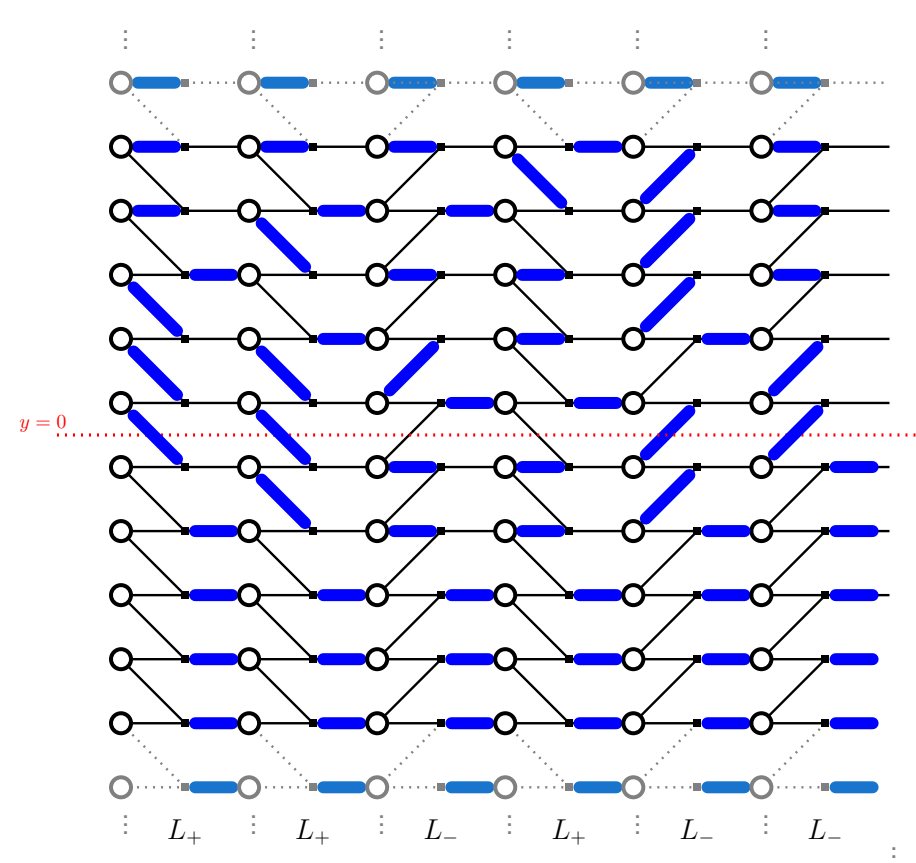

FigURE 12. A rail yard graph with a constant LR sequence $\underline{a}=L^{6}$ and sign sequence $\underline{b}=++-+--$, equipped with a pure covering.

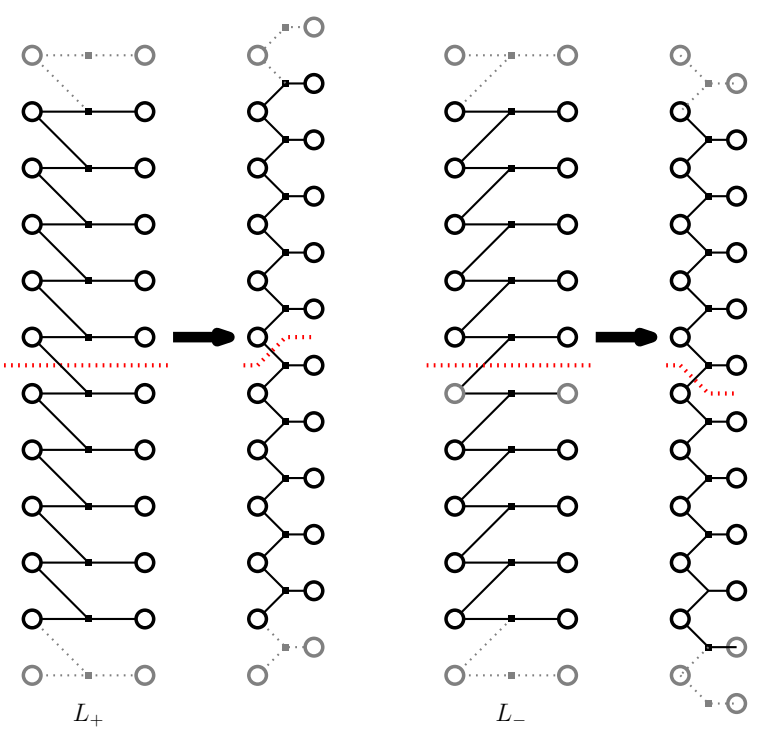

FiguRE 13. Deformations of the elementary RYG of type $L+$ and $L$ - that generate a "honeycomb" lattice by concatenation.

sequence $\underline{b}$, see Figure 15 again. Note also that all the lozenge tilings corresponding to pure dimer coverings of $\operatorname{RYG}(\ell, r, \underline{a}, \underline{b})$ are obtained by "adding cubes" to this 3 -dimensional diagram in such a way that the heights of cubes stay nonincreasing on horizontal coordinates, going away from the axes. In particular the case $\underline{b}=$ $+{ }^{n}{ }^{n}$ corresponds to plane partitions of half-width at most $n$. This 3 -dimensional interpretation is well known and is the one already considered in OR03. 


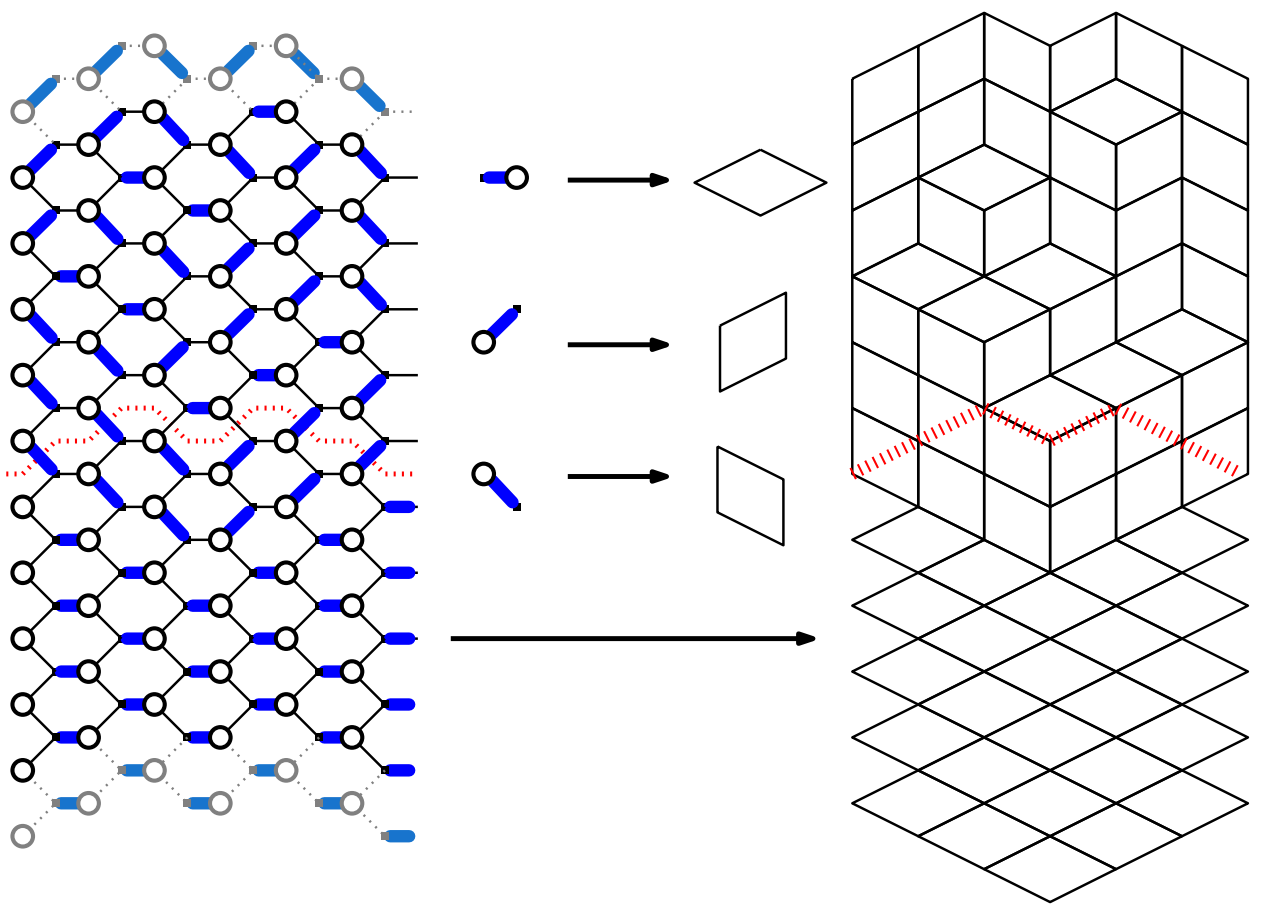

FiguRE 14. Left: The dimer covering of Figure 12 as a covering of the honeycomb lattice, via the transformations of Figure 13 . Right: The same objects displayed as a "lozenge tiling" of the plane.

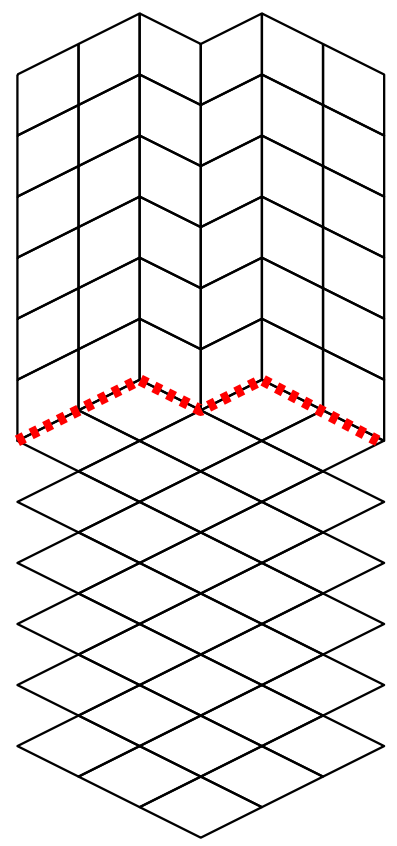

Figure 15. The fundamental lozenge tiling corresponding to the case $\underline{a}=L^{6}$ and $\underline{b}=++-+--$. 
5.2. Domino tilings. We now consider the case where the sequence $\underline{a}$ has even length and is an alternation of $L$ and $R$. Up to an elementary symmetry we can assume that $\underline{a}=(L R)^{k}$, where $r-\ell+1=2 k$, and we fix an arbitrary sign sequence $\underline{b} \in\{+,-\}^{2 k}$. It follows from a simple inspection of the face types of elementary rail yard graphs (Figure 8(a)) that all the inner faces of the graph $\operatorname{RYG}(\ell, r, \underline{a}, \underline{b})$ have degree 4 or 8 . Moreover, for $0 \leq i<k$, odd vertices located at the abscissa $x=-2 \ell-1+4 i+2$ all have degree 2 (since they lie at the interface between an $L$-type and $R$-type elementary graphs, from left to right). These degree 2 vertices are bounded by faces of degree 8 on their two sides. See Figure 16 for an example.

We now let $\tilde{R}(\ell, r, \underline{a}, \underline{b})$ be the graph obtained by contracting all the edges incident to these inner vertices of degree 2: in this graph all inner vertices of degree 2 have disappeared, and all the non-boundary faces have degree 4, see Figure 17-Left for an example. Moreover, it is easy to see that each dimer covering of $\operatorname{RYG}(\ell, r, \underline{a}, \underline{b})$ induces a dimer covering of $\tilde{R}(\ell, r, \underline{a}, \underline{b})$, with the same boundary conditions (just forget dimers on contracted edges and leave the other dimers as they were). The coordinate transformation on non deleted vertices that goes from $\mathrm{RYG}(\ell, r, \underline{a}, \underline{b})$ to $\tilde{R}(\ell, r, \underline{a}, \underline{b})$ is given by:

$$
(-2 \ell-1+x, y) \longmapsto\left(-2 \ell-1+x-2\left\lfloor\frac{x+2}{4}\right\rfloor, y\right) .
$$

Since all the inner vertices and faces in this new graph have degree 4, this new graph is isomorphic to a portion of the square lattice. We let the reader check that this isomorphism can be made explicit by composing 85 with the transformation:

$$
(-2 \ell-1+x, y) \longmapsto\left(-2 \ell-1+x+y-K_{x}, y-K_{x}\right),
$$

where $K_{x}=\sum_{i=1}^{x}(-1)^{i+b_{i}}$. See Figure 17 for an explicit example. The image of $\tilde{R}(\ell, r, \underline{a}, \underline{b})$ via these transformations is a portion of the square lattice lying in the oblique strip $\{(X, Y):|Y-X+2 \ell+1| \leq 2 k\}$, see again Figure 17 .

Similarly as in Section 5.1, we can dualize this picture to switch between a description in terms of dimers to one in terms of tilings. The dual of the square lattice is again the square lattice, and any dimer in the primal induces a domino (the union of two adjacent squares) in the dual. We thus recover a model of tilings of the oblique strip by dominos, which are exactly the steep tilings introduced in [BCC14]. We invite the reader to consult this reference for a thorough discussion on steep tilings, their link with height functions, their encoding in terms of partitions, etc. Here we just mention again that, not only do we recover here the enumerative results already proved in $\mathrm{BCC} 14$, but we obtain the inverse Kasteleyn matrix and dimer correlations for these models, up to the changes of coordinates described above.

Two particular subclasses of steep tilings had been considered previously. The first one is the class of domino tilings of the Aztec diamond, which corresponds to the case where the sequence $\underline{b}$ is also alternating. As far as we know, this is the only case for which the inverse Kasteleyn matrix has been computed before Hel00, CY14. The other one is given by pyramid partitions You09, that correspond to the case where $\underline{b}=+^{\infty}{ }^{\infty}$. See [BCC14, Section 4.2] for their connection with steep tilings. We leave as an exercise the task of making fully explicit the changes of coordinates and the calculations for pyramid partitions, similarly as we will do for the Aztec diamond in the next section.

Remark 20. Going from rail yard graphs to steep tilings induces, in a sense, no loss of generality. Indeed, by forbidding diagonal dimers in the column $i$ of a rail yard graph (by taking their weight $x_{i}$ to be zero), this column becomes trivial in the sense that its two boundary states are necessarily equal, and hence it can 


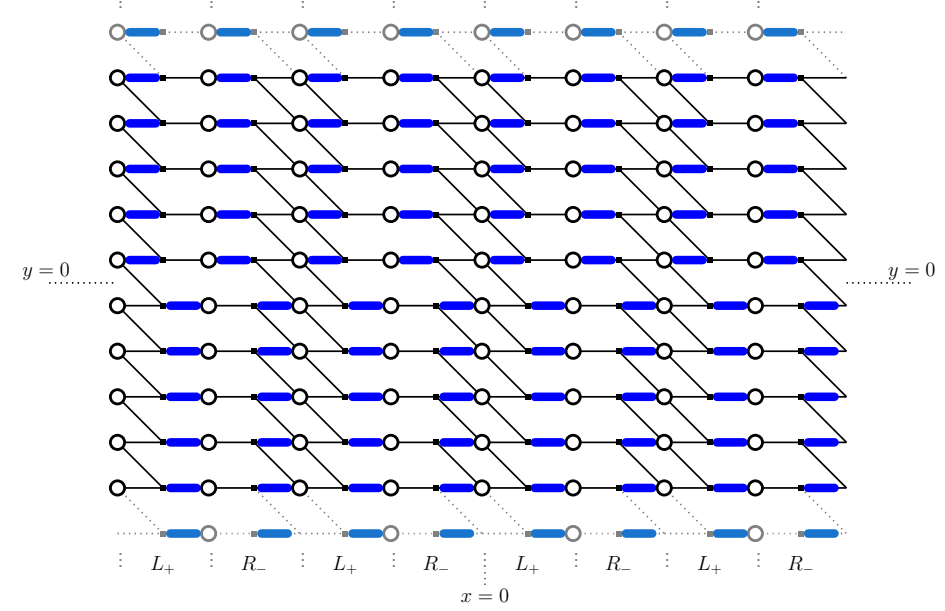

FIGURE 16. A rail yard graph with LR sequence $\underline{a}=(L R)^{n}$ and sign sequence $\underline{b}=(+-)^{n}$ for $n=4$, equipped with its fundamental dimer covering.

be contracted in the graph (i.e. we can drop the corresponding elements from the LR and sign sequences). Starting from an infinite alternating LR sequence, it is possible to produce any LR sequence by performing such contractions, and hence to obtain any RYG. The coordinates of rail yard graphs however allow to express the dimer correlations in a more compact form, which is one of our motivations for introducing them.

\section{The Aztec diamond Revisited}

In order to illustrate Theorem 5 on a concrete example, we concentrate in this section on the particular case of domino tilings of the Aztec diamond of size $n$ [EKLP92a, EKLP92b], which can be obtained as a rail yard graph associated with the sequences $\underline{a}=(L R)^{n}$ and $\underline{b}=(+-)^{n}$, see Figures 16 and 17 We will suppose that $\ell=0$ and $r=2 n-1$, and denote by $G_{n}$ the corresponding rail yard graph. Note that, though $G_{n}$ is an infinite graph, its admits only a finite number $\left(2^{n(n+1) / 2}\right)$ of pure dimer coverings. Those pure dimer coverings coincide with the fundamental covering outside a finite region which is isomorphic to the Aztec diamond graph of size $n$, see [BCC14, Section 4.1] for a discussion of this phenomenon in the language of steep tilings. The Aztec diamonds are essentially the only RYGs having a finite number of pure dimer configurations (this can be seen from the enumerative results of Section 2.4 and Remark 20 .

Let us now discuss the probability distributions over domino tilings of the Aztec diamond that we are considering. Recall the definition (1) of the weight of a configuration in the multivariate RYG dimer model. For a generic sequence $x_{0}, \ldots, x_{2 n-1}$, we obtain the so-called Stanley weighting scheme [Pro97, Yan91, see also BCC14, Remark 2]. The partition function reads

$$
Z\left(G_{n}, \underline{x}\right)=\prod_{\substack{0 \leq i<j \leq 2 n-1 \\ i \text { even, } j \text { odd }}}\left(1+x_{i} x_{j}\right)
$$

which is a polynomial in the $x$ 's. By specialization we obtain the following distributions considered originally in EKLP92a]:

- the uniform distribution, obtained by taking $x_{i}=1$ for all $i$, 

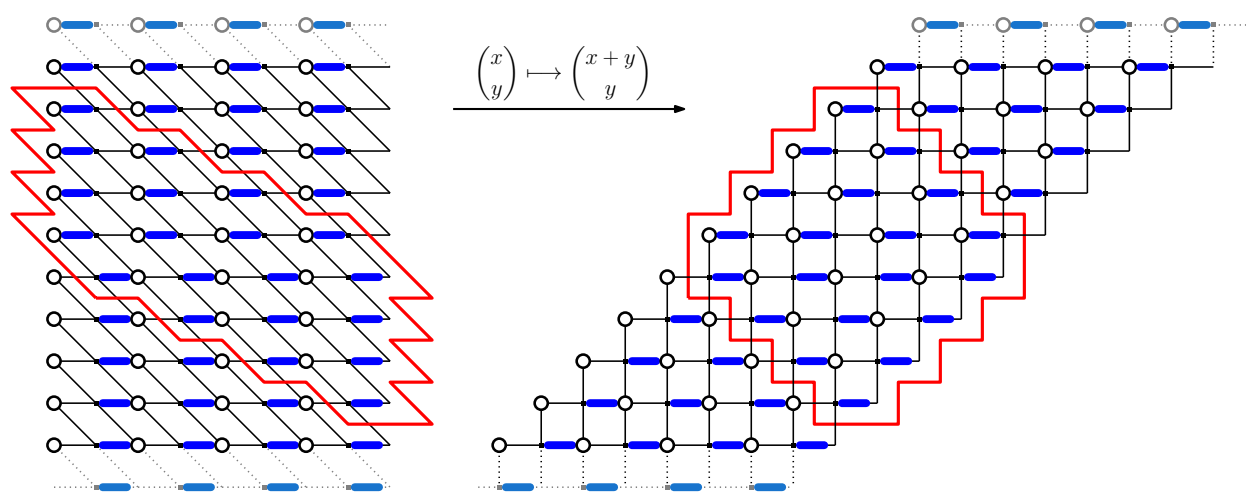

FiguRE 17. Left: The graph obtained by contracting all the vertices of degree 2 in the RYG of Figure 16, that is, all the vertices of abscissa congruent to 2 mod 4. Right: the image of the graph on the left by the linear transformation $\left(\begin{array}{l}x \\ y\end{array}\right) \mapsto\left(\begin{array}{c}x+y \\ y\end{array}\right)$. The graph is a portion of a square lattice of mesh 1 . On both sides, the region of the graph where a covering may differ from the fundamental one is represented in red. One recognizes the shape of the Aztec diamond of size $n=4$. Note that the coordinate transformation on uncontracted vertices to go directly from the graph of Figure 16 to the graph on the right is given by $\left(\begin{array}{l}x \\ y\end{array}\right) \mapsto\left(\begin{array}{c}\phi(x)+y \\ y\end{array}\right)$ where $\phi(x)=x-2\left\lfloor\frac{x+2}{4}\right\rfloor$ if $x \geq 0$ and $\phi(-x)=-\phi(x)$.

- the biased distribution, obtained by taking $x_{i}=1$ for $i$ even and $x_{i}=\lambda>0$ for $i$ odd (or equivalently $x_{i}=\sqrt{\lambda}$ for all $i$ ): this corresponds to attaching a weight $\lambda$ to each pair of diagonal dimers (which become vertical dominos in the Aztec diamond picture),

- the so-called $q^{\mathrm{vol}}$ distribution, obtained by taking $x_{i}=q^{i}$ for $i$ odd and $x_{i}=q^{-i}$ for $i$ even (which is the $q$-RYG specialization), and more generally the biased $q^{\text {vol }}$ distribution, obtained by taking $x_{i}=\lambda q^{i}$ for $i$ odd and $x_{i}=q^{-i}$ for $i$ even.

Let us mention that our present approach does not seem to apply to the twoperiodic weighting considered in [CY14, Section 6], nor to the weightings considered in DFSG14.

The study of correlations in domino tilings of the Aztec diamond has been a popular topic (especially among the members of the "domino forum") and there are many previously known results, published or unpublished. In the rest of this section, we rederive several of these results as consequences of our general Theorem 5

6.1. The biased creation rate and edge-probability generating function. Let us consider the Aztec diamond of size $n$ in the natural coordinates with the origin at the center. We are interested in the probability to find a domino of a given type at a given position, under the biased distribution. Recall that we may distinguish four types of dominos: north-, south-, west- and east-going CEP96. By symmetry it is enough to consider only one type of domino, and we denote by $P_{\lambda}(x, y, n)$ the probability that $(x-1 / 2, y)$ is the center of a west-going domino in a biased random tiling of the Aztec diamond of size $n$. Due to parity constraints, this probability vanishes unless $x$ and $y$ are integers such that $x+y+n$ is odd. As apparent from Figures 16 and 17, west-going dominos correspond in the RYG setting to diagonal dimers in columns of type $L+$ (which makes them easier than 
north-going dominos to deal with, since there can be no spurrious diagonal dimers outside the "interesting" region).

Getting an expression for $P_{\lambda}(x, y, n)$ (or its analogues for other domino orientations) ameneable to asymptotic analysis has been of interest to several people, for instance it is used in CEP96 as a way to proving the arctic circle theorem and its generalization to arbitrary $\lambda$. However, this paper states the required expressions without proofs, and refers instead to a preprint by Gessel, Ionescu and Propp that has not appeared so far. The proof for $\lambda=1$ (uniform distribution) can be found in Helfgott's senior thesis [Hel00]. More recently, an expression for the related generating function (still for $\lambda=1$ only) was proved by two methods in Du's master thesis [Du11, and was used in [BP11] as yet another route to the arctic circle theorem (we note that those two references mention another lost "DGIP" preprint). At the suggestion of James Propp, which we thank for pointing out this gap in the literature, we will explain how expressions for $P_{\lambda}(x, y, n)$ (for general $\lambda$ ) can be obtained as applications of our Theorem 5 .

The first step consists in going from dominos to dimers on RYGs. Using the identification discussed above, a west-going domino centered on $(x-1 / 2, y)$ corresponds to a dimer on the edge $(\alpha, \beta)$ with

(88) $\alpha=(2 m, y-1 / 2), \quad \beta=(2 m-1, y+1 / 2), \quad m=\frac{n+x-y+1}{2} \in[1 . . n]$.

By Theorem 5 we immediately deduce the expression

$$
\begin{aligned}
& P_{\lambda}(x, y, n)= \\
& \quad \frac{1}{(2 i \pi)^{2}} \oint_{C_{z}} \oint_{C_{w}}\left(\frac{1-w}{1-z}\right)^{m}\left(\frac{1+\lambda / w}{1+\lambda / z}\right)^{n+1-m} \frac{(w / z)^{y}}{(z-w)(1-w)} d z d w
\end{aligned}
$$

where $C_{z}$ is a positively oriented contour containing 0 and $-\lambda$ in its interior, but not 1 , and $C_{w}$ is a positively oriented contour containing $C_{z}$ in its interior. Simpler expressions can be obtained for two related quantities: the so-called biased creation rate

$$
\mathrm{Cr}_{\lambda}(x, y, n)=\frac{\lambda+1}{\lambda}\left(P_{\lambda}(x, y, n)-P_{\lambda}(x+1, y, n-1)\right)
$$

and the edge-probability generating function

$$
\Pi_{\lambda}(u, v, t)=\sum_{x, y, n} P_{\lambda}(x, y, n) u^{x} v^{y} t^{n} .
$$

Note that, in CEP96, the bias $p$ is related to our $\lambda$ by $p=1 /(1+\lambda)$, and the biased creation rate is expressed in terms of north-going dominos hence the present definition is adapted to the case of west-going dominos.

Let us first consider the biased creation rate. Taking the difference $P_{\lambda}(x, y, n)-$ $P_{\lambda}(x+1, y, n-1)$ in the double contour integral (89), the integrand is multiplied by a factor $1-\frac{1+\lambda / z}{1+\lambda / w}=\frac{\lambda(z-w)}{z(w+\lambda)}$, leading to a cancellation of the denominator $(z-w)$, hence to the factorization

$$
\begin{aligned}
\operatorname{Cr}_{\lambda}(x, y, n)= & (1+\lambda)\left(\oint_{C_{z}}(1-z)^{-m}(z+\lambda)^{-(n+1-m)} z^{n+1-m-y} \frac{\mathrm{d} z}{2 i \pi z}\right) \\
& \times\left(\oint_{C_{w}}(1-w)^{m-1}(w+\lambda)^{n-m} w^{y+m-n} \frac{\mathrm{d} w}{2 i \pi w}\right) .
\end{aligned}
$$

Note that, by Cauchy's residue formula, the second integral is equal to the coefficient of $w^{n-m-y}$ in $(1-w)^{m-1}(w+\lambda)^{n-m}$, which is a Krawtchouk polynomial. The first integral is of a similar nature, except that the role of the zeros $\{1,-\lambda\}$ and of the 
poles $\{0, \infty\}$ in the integrand are exchanged. This suggests to perform the change of variables $z=\frac{1-u}{1+\lambda^{-1} u}$ which transforms the first integral in $(92)$ into:

$$
(1+\lambda)^{-n} \lambda^{m-1} \oint_{C_{u}} u^{1-m}\left(1+\lambda^{-1} u\right)^{m+y-1}(1-u)^{n-m-y} \frac{-d u}{2 i \pi u},
$$

where $C_{u}$ is a small negatively oriented contour encircling $u=0$ (note that the image of the negatively oriented contour $C_{u}$ under the change of variable $z=\frac{1-u}{1+\lambda^{-1} u}$ is a negatively oriented contour encircling $z=1$, which is homotopic in $\mathbb{C} \cup\{\infty\} \backslash$ $\{1,-\lambda\}$ to the positively oriented contour $C_{z}$ ). Using Cauchy's residue formula, the second integral is equal to the coefficient of $u^{m-1}$ in $\left(1+\lambda^{-1} u\right)^{m+y-1}(1-u)^{n-m-y}$. Redistributing powers of $\lambda$ we finally obtain:

Proposition 21. The biased creation rate reads

$$
\mathrm{Cr}_{\lambda}(x, y, n)=\left(\frac{\lambda}{1+\lambda}\right)^{n-1} c_{\lambda}(A, B, n-1) c_{\lambda}(B, A, n-1)
$$

where $A=n-m-y=(n-1-x-y) / 2, B=m-1=(n-1+x-y) / 2$ and $c_{\lambda}(A, B, n)$ is the coefficient of $z^{A}$ in $(1-z)^{B}\left(1+\lambda^{-1} z\right)^{n-B}$.

Note that $\frac{\lambda}{1+\lambda}=(p-1)$ and $\lambda^{-1}=\frac{p}{1-p}$, so we recover [CEP96, Proposition 23], up to the exchange $p \mapsto 1-p$ and the antidiagonal reflection $(x+y, x-y) \mapsto$ $(-x-y, x-y)$ that correspond to the fact that we consider west-going rather than north-going dominos. [CEP96, Proposition 2] also follows, by taking $\lambda=1$, i.e. $p=1 / 2$.

We now turn to the edge-probability generating function. We need to multiply (89) by $u^{x} v^{y} t^{n}=(t u)^{m}(t / u)^{n+1-m}(u v)^{y} / t$ and sum over all $n \geq 0, m \in[1 . . n]$ and $y \in \mathbb{Z}$. Assuming that $\lambda<1, C_{z}$ and $C_{w}$ can be taken as circles with center 0 and radiuses between $\lambda$ and 1 . For $t$ small enough and $u, v$ of modulus close to 1 , it is possible to interchange the double sum over $n, m$ and the double contour integral to yield

$$
\Pi_{\lambda}(u, v, t)=\sum_{y \in \mathbb{Z}} \frac{1}{(2 i \pi)^{2}} \oint_{C_{z}} \oint_{C_{w}} \phi(z, w)(u v w / z)^{y} \frac{d z}{z} \frac{d w}{w}
$$

where

$$
\phi(z, w)=\frac{\frac{t u(1-w)}{1-z}}{1-\frac{t u(1-w)}{1-z}} \cdot \frac{\frac{t(1+\lambda / w)}{u(1+\lambda / z)}}{1-\frac{t(1+\lambda / w)}{u(1+\lambda / z)}} \cdot \frac{z w}{t(z-w)(1-w)} .
$$

The sum over all $y$ allows to get rid of one integral. More precisely, assuming $|u v|<1$ and $|w|$ fixed, we take two different contours $C_{z}$ depending on the sign of $y$, namely a circle $C_{z}^{+}$(resp. $C_{z}^{-}$) of radius slightly larger (resp. smaller) than $|u v w|$ if $y \geq 0$ (resp. $y<0$ ). Splitting the sum over $y$ in two accordingly, we may interchange each sum with the integral, resulting in

$$
\begin{aligned}
\Pi_{\lambda}(u, v, t) & =\frac{1}{(2 i \pi)^{2}} \oint_{C_{w}}\left(\left(\oint_{C_{z}^{+}}-\oint_{C_{z}^{-}}\right) \frac{\phi(z, w)}{z-u v w} d z\right) \frac{d w}{w} \\
& =\frac{1}{2 i \pi} \oint_{C_{w}} \phi(u v w, w) \frac{d w}{w} .
\end{aligned}
$$

In the latter integral, the integrand has two poles, but only one of them falls within $C_{w}$ for $t$ small and $|u v|$ close to 1 , and we end up with:

Proposition 22. The biased edge-probability generating function reads

$$
\Pi_{\lambda}(u, v, t)=\frac{\lambda t}{(1-t / u)\left((1+\lambda)\left(1+t^{2}\right)-t\left(u+u^{-1}\right)-\lambda t\left(v+v^{-1}\right)\right)} .
$$


For $\lambda=1$, we recover the expression given in Du11, BP11] (again up to the change of variables needed to pass from west-going to north-going dominos).

Remark 23. The generating function associated with the biased creation rate takes a much more symmetric form, namely

$$
\begin{aligned}
\sum_{x, y, n} \operatorname{Cr}_{\lambda}(x, y, n) u^{x} v^{y} t^{n} & =\frac{\lambda+1}{\lambda}(1-t / u) \Pi_{\lambda}(u, v, t) \\
& =\frac{(\lambda+1) t}{\left((1+\lambda)\left(1+t^{2}\right)-t\left(u+u^{-1}\right)-\lambda t\left(v+v^{-1}\right)\right)},
\end{aligned}
$$

and it remains the same for other types of dominos. The combinatorial explanation of this phenomenon (and of the meaning of the term "creation rate") comes from the domino shuffling algorithm [EKLP92b, which implies that $\operatorname{Cr}_{\lambda}(x, y, n)$ is the probability that, in a biased random tiling of the Aztec diamond of size $n$, the $2 \times 2$ square centered at $(x, y)$ is covered by exactly two dominos (regardless of their orientation).

6.2. The inverse Kasteleyn matrix. Chhita and Young gave in CY14, Section 5] a formula for the inverse Kasteleyn matrix of the Aztec diamond of size $n$, for the biased $q^{\text {vol }}$ distribution (whose definition is recalled on page 33). Under this distribution the probability for a tiling is proportional to $q$ to the number of flips from the fundamental configuration times $\sqrt{\lambda}$ to the number of vertical dominos. We now explain how to relate their formula with the entries $\mathcal{C}_{\alpha, \beta}$.

Let $\alpha=\left(\alpha^{\mathrm{x}}, \alpha^{\mathrm{y}}\right)$ be an even vertex and $\beta=\left(\beta^{\mathrm{x}}, \beta^{\mathrm{y}}\right)$ an odd vertex, which have survived the contraction of edges. The coordinates we will use are those of the contracted graph, so $\alpha^{\mathrm{x}} \in[0 . .2 n-2]$ and $\beta^{\mathrm{x}} \in[-1 . .2 n-1]$.

In this particular case, for $k \in[-1 . .2 n-1]$, the function $F_{k}(z)$ from Equation (8) becomes:

$$
F_{k}(z)=\frac{1}{\prod_{j=0}^{\lfloor k / 2\rfloor}\left(1-\sqrt{\lambda} q^{-2 j} z\right) \prod_{j=\lfloor(k+1) / 2\rfloor}^{n-1}\left(1+\sqrt{\lambda} q^{2 j+1} / z\right)} .
$$

Chhita and Young use coordinates $\left(x_{1}, x_{2}\right)$ (resp. $\left.\left(y_{1}, y_{2}\right)\right)$ to localize odd (resp. even) vertices. In their terminology, they are white and black respectively. These coordinates correspond to axes that are along the diagonals of Figure 17 on the right.

The correspondence between the two systems of coordinates is

$$
\left\{\begin{array}{l}
x_{1}=1+\beta^{\mathrm{x}}+2 \beta^{\mathrm{y}} \\
x_{2}=1+\beta^{\mathrm{x}}
\end{array}, \quad\left\{\begin{array}{l}
y_{1}=1+\alpha^{\mathrm{x}}+2 \alpha^{\mathrm{y}} \\
y_{2}=1+\alpha^{\mathrm{x}}
\end{array} .\right.\right.
$$

Performing the change of variable

$$
\zeta=-\frac{q^{y_{2}-2}}{w}, \quad \theta=-\frac{q^{y_{2}-2}}{z},
$$

in the integral defining $K_{\text {col }}^{-1}$ in [CY14, Theorem 5.1] in the case when $x_{2} \leq y_{2}$, one recovers the same factors for the rational fraction in $\zeta$ and $\theta$ as for $G_{\alpha, \beta}(\zeta, \theta)$, up to possibly numerical multiplicative constants. One has just to check that the contours enclose the same sets of poles. Under the change of variables, the contour $\Gamma_{1, q}$ becomes a contour for $\zeta$ enclosing $-\sqrt{\lambda} q^{y_{2}},-\sqrt{\lambda} q^{y_{2}+2}, \ldots,-\sqrt{\lambda} q^{2 n-1}$ (and which may or may not enclose zero, since the original rational fraction in $w$ is regular at infinity), and the contour $\Gamma_{0}$ is mapped to a large contour for $\theta$ containing separating infinity from a domain containing all the poles and the contour for $\theta$. This contour can be deformed freely as long as it does not cross the one for $\zeta$ or infinity, because $1 / F_{k}(\theta)$ is a polynomial, and thus has no poles. 
In the case when $x_{2}>y_{2}$, the extra term in CY14 comes from the residue at $w=z$, which can be integrated to the double integral, at the cost of interchanging the nesting of the contours.

6.3. The arctic circle theorem. Under the uniform measure, domino tilings of a large Aztec diamond exhibit a spatial phase transition, known as the arctic circle phenomenon JPS98, Joh05]. Outside the inscribed circle, with probability exponentially close to 1 , all dominos are arranged in a brickwall fashion. This is called the frozen region. Inside the circle, the probability of each orientation is non degenerate and does not go to 0 or 1 . We now explain how to recover this phenomenon from our formalism. The parameters $q$ and $\lambda$ are now set to 1 .

Let $\alpha=\left(\alpha^{\mathrm{x}}, \alpha^{\mathrm{y}}\right)=\left(2 m, y-\frac{1}{2}\right)$ be an even vertex. The probability $\rho_{\alpha}$ that a dimer connects $\alpha$ with $\beta=\left(\beta^{\mathrm{x}}, \beta^{\mathrm{y}}\right)=\left(2 m-1, y+\frac{1}{2}\right)$ is given by 89 with $\lambda=1$. In the scaling limit $n \rightarrow \infty, m / n \rightarrow \tau, y / n \rightarrow \chi$, this probability becomes

$$
\rho_{\alpha}=\oint_{C_{z}} \oint_{C_{w}} \exp (n(S(z, \tau, \chi)-S(w, \tau, \chi)+o(1))) \frac{\mathrm{d} z \mathrm{~d} w}{(z-w)(2 i \pi)^{2}}
$$

where

$$
S(z, \tau, \chi)=-\tau \log (1-z)-(1-z) \log (1+1 / z)-\chi \log (z) .
$$

We now proceed as in OR03 to obtain the asymptotics of this probability. For fixed $(\tau, \chi)$, the function $z \mapsto S(z, \tau, \chi)$ has two critical points.

- If the two critical points are real, the integral goes to 0 or 1 exponentially fast with $n$ by the saddle point method. The point $(\tau, \chi)$ is in the frozen region.

- If the two critical points are complex conjugate, one can move the contours so that they cross transversally at the complex critical points to apply again the saddle point method. By doing so, we pick the contribution of the residue at $z=w$, which gives the main contribution of the integral, giving a result strictly between 0 and 1 .

The transition between those two regimes correspond to the value of $(\tau, \chi)$ for which the two critical points merge. This happens when the discriminant of the numerator of $\partial S(z, \tau, \chi) / \partial z$ is equal to zero. This gives

$$
(2 \tau-1)^{2}-4(\tau+\chi)(1-\chi-\tau)=0,
$$

which under the change of variables

$$
\left\{\begin{array}{l}
u=2 \tau+\chi \\
v=\chi
\end{array}\right.
$$

corresponds to the circle $2(u-1)^{2}+2 v^{2}=1$ inscribed in the limiting square of the Aztec diamond, given by $|u-1|+|v| \leq 1$.

\section{Conclusion}

We have introduced the rail yard graph dimer model, and computed its partition function and correlation functions. We point out that it corresponds essentially to the most general Schur process with nonnegative transition probabilities, see the discussion in Bor11, Sections 1 and 2]: we handle an arbitrary finite number of " $\alpha$ " and " $\beta$ " specializations, and any other specialization can be obtained by taking suitable limits (in particular, to get the Poissonized Plancherel measure, one shall consider the "dilute" limit of RYGs, namely take a sign sequence of the form $+^{n}{ }^{n}$, an arbitrary LR sequence, and a constant weight sequence $z / n$, then let $n \rightarrow \infty$ ).

Many directions can be explored from here. By applying the random generation algorithms of $\left[\mathrm{BBB}^{+} 14\right]$, we may generate large RYG dimer configurations, which 
allows to observe limit shape phenomena as in the cases of (skew) plane partitions OR03, OR07 and of the Aztec diamond, discussed above. RYG seem to allow for an even larger variety of singular points on limit shapes, and of corresponding limiting processes, which are currently under investigation.

The appearance of the rational edge-probability generating function $(98)$ in the context of the Aztec diamond (when summing over diamonds of all sizes) raises the question whether such rationality phenomenon may subsist for other types of RYGs. A natural idea is to consider RYGs with periodic LR, sign and weight sequences. Preliminary research indicates the rationality phenomenon occurs only in another case, namely skew plane partitions of "staircase" shape. In other cases, we obtain an algebraic, but not rational, generating function (algebraicity being expected from the very nature of our computations).

In this paper we have obtained the correlations for pure RYG dimer configurations (by computing vacuum-to-vacuum expectation values of fermionic operators). Other types of boundary conditions can be considered, as in BCC14 where the corresponding partition functions were computed (and the extension to RYGs is straightforward). However, adapting the computation of correlation functions done in the present paper is not so easy, since it requires an adaptation of Wick's formula. For arbitrary but fixed boundary conditions, we know from general facts, namely the generalized Wick theorem [AZ13] or the Eynard-Mehta theorem BR05], that correlations will still be of determinantal form, however it is not clear how to compute explicitly the propagator/determinantal kernel. Such computation could be done by Petrov Pet14 for some lozenge tilings, and we are looking for other tractable cases. Also of interest is the case of free boundary conditions (that is, we sum over all possible boundary states). When only one of the boundaries of the RYG is free (corresponding to symmetric RYG dimer configurations), the correlations are known to be Pfaffian BR05, and we have found an adaptation of Wick's formula which would allow for a computation similar to that done in the present paper, bypassing the use of the (Pfaffian analogue of) Eynard-Mehta's theorem. Details should appear in a subsequent publication, see also [DFR12, Vul13, Pan14] for related results. When the two boundaries of the RYG are free, the nature of the correlations is unknown, even though the partition function can be computed following the lines of BCC14. We believe they should be the Pfaffian analogues of correlations for RYGs with periodic boundary conditions, related to the periodic Schur process of Bor07.

Finally, a tantalizing question is whether it is possible to consider "interacting" deformations of our dimer models. Besides the directions mentioned in the conclusion of BCC14, let us mention that fermionic techniques have been recently used, together with methods from constructive field theory, to prove rigorous results about interacting dimer models, see e.g. [GMT14] and references therein. Another intriguing fact is that identities arising from $z$-measures (which are instances of Schur measures) have found applications in the context of quantum integrable systems $\mathrm{KKM}^{+} 11$.

\section{ACKNOWLEDGMents}

We would like to thank Dan Betea, Alexei Borodin, Sunil Chhita, Philippe Di Francesco, Patrik Ferrari, Jean-Michel Maillet, Richard Kenyon, Leo Petrov, Senya Shlosman and Mirjana Vuletić, for their constructive comments and helpful discussions. 


\section{Appendix A. Commutation of Bosonic And Fermionic operators}

In this section, we give a self-contained combinatorial proof of the commutation relations between the bosonic and the fermionic operators stated in Proposition 11 .

Proof. Let us first prove (37). It is equivalent to prove that for any $k$ and for any $\lambda$,

$$
\Gamma_{R+}(x) \psi_{k}|\lambda\rangle=\left(\psi_{k}+x \psi_{k-1}\right) \Gamma_{R+}(x)|\lambda\rangle
$$

Define

$$
n_{k}=\#\left\{j>k, \lambda_{j}=\bullet\right\} .
$$

Case 1 : $\lambda$ has a white marble in position $k$.

$\Gamma_{R+}(x) \psi_{k}|\lambda\rangle$ enumerates the admissible dimer covers of an elementary rail yard graph of type $(R,+)$ and with right boundary equal to $\psi_{k}|\lambda\rangle$. Each dimer cover is specified by the value $\mu$ of the left boundary. Here,

$$
\psi_{k}|\lambda\rangle=(-1)^{n_{k}}\left|\lambda^{(k)}\right\rangle
$$

so the vertex in position $k$ on the right boundary has to be incident to a certain edge $e_{k}$ in the dimer cover. Thus $\Gamma_{R+}(x) \psi_{k}|\lambda\rangle$ is a sum of two types of terms, the first (resp. second) type corresponds to $e_{k}$ horizontal (resp. diagonal).

By the same argument as for the localization of horizontal dimers on a "double" column, dimer covers of the first type are in bijection with (and have the same weight as) dimer covers with right boundary $\lambda$ and with left boundary $\mu^{(k)}$. Such covers are enumerated by $\Gamma_{R+}(x)|\lambda\rangle$. To obtain the original left boundary, $\mu$, from this new cover, we need to apply $\psi_{k}$ to $\Gamma_{R+}(x)|\lambda\rangle$. The sign appearing is again $(-1)^{n_{k}}$, because the number of black marbles above position $k$ is the same in $\mu^{(k)}$ as in $\lambda$. So the first term in the sum is equal to

$$
\psi_{k} \Gamma_{R+}(x)|\lambda\rangle \text {. }
$$

By using the bijection used to localize diagonal dimers, and observing that the weights differ by a factor $x$, we obtain that the second term of the sum is equal to

$$
x \psi_{k-1} \Gamma_{R+}(x)|\lambda\rangle .
$$

Note that the signs cancel out correctly because the number of black marbles above position $k-1$ on the left boundary is equal to the number of black marbles above position $k$ on the right boundary.

Thus we conclude in case 1 .

Case 2: $\lambda$ has a black marble in position $k$.

Here, $\psi_{k}|\lambda\rangle=0$, so the left-hand side of (107) vanishes.

$\Gamma_{R+}(x)|\lambda\rangle$ enumerates the admissible dimer covers of an elementary rail yard graph of type $(R,+)$ and with right boundary equal to $\lambda$. Each dimer cover is specified by the value $\mu$ of the left boundary. The vertex in position $k$ on the right boundary has to be incident to a certain edge $e_{k}$ in the dimer cover. Thus $\Gamma_{R+}(x) \psi_{k}|\lambda\rangle$ is a sum of two types of terms, the first (resp. second) type corresponds to $e_{k}$ horizontal (resp. diagonal).

If $\mu$ is a term of the first type, $\mu$ has a black marble in position $k$, thus $\psi_{k}+$ $x \psi_{k-1}|\mu\rangle=x \psi_{k-1}|\mu\rangle$ and

$$
x \psi_{k-1}|\mu\rangle= \begin{cases}x(-1)^{n_{k}+1} \mu^{(k-1)} & \text { if } \mu \text { has a white marble in position } k-1 \\ 0 & \text { otherwise }\end{cases}
$$

The sign is due to the fact that the number of black marbles in $\mu$ above the position $k-1$ is equal to the number of black marbles in $\lambda$ above the position $k$, to which we must add the black marble in $\lambda$ in position $k$. 
If $\mu$ is a term of the second type, $\mu$ has a black marble in position $k-1$, thus $\psi_{k}+x \psi_{k-1}|\mu\rangle=\psi_{k}|\mu\rangle$ and

$$
\psi_{k}|\mu\rangle= \begin{cases}(-1)^{n_{k}} \mu^{(k)} & \text { if } \mu \text { has a white marble in position } k \\ 0 & \text { otherwise. }\end{cases}
$$

So all the nonzero terms of $\left(\psi_{k}+x \psi_{k-1}\right) \Gamma_{R+}(x)|\lambda\rangle$ have black marbles in positions $k-1$ and $k$, and each term appears twice, with the same weight (because if $\mu$ is a term of the second type in $\Gamma_{R+}(x)|\lambda\rangle$, it already carries a factor $x$ coming from the diagonal dimer $e_{k}$ ) and with opposite sign.

Thus the right-hand side of (107) also vanishes.

This concludes the proof of (37). Formula (41) is proved similarly, replacing $\psi_{k-1}$ by $\psi_{k+1}$ and $z$ by $\frac{1}{z}$.

We noted that $\Gamma_{R+}(x)$ was conjugated to $\Gamma_{L+}(x)$ via $\omega$. Observe now that $\psi_{l}^{*}$ is conjugated to $\psi_{l}$ via $\omega$, up to a $\operatorname{sign}(-1)^{s_{l}}$ verifying $(-1)^{s_{l+1}}=-(-1)^{s_{l}}\left(s_{l}\right.$ is defined by an equation analogous to (35). This enables us to deduce formulas (38) and 42 .

Using that $\Gamma_{R+}(x), \Gamma_{L+}(x)$ and $\psi(z)$ are respectively adjoint of $\Gamma_{R-}(x), \Gamma_{L-}(x)$ and $\psi(1 / z)$, we deduce the last four formulas.

\section{APPENDix B. WiCK's FORMUlA}

In this section, we provide a proof of the identity

$$
\left\langle\emptyset\left|\mathcal{T}\left(\Psi\left(\alpha_{1}\right), \Psi^{*}\left(\beta_{1}\right), \ldots, \Psi\left(\alpha_{s}\right), \Psi^{*}\left(\beta_{s}\right)\right)\right| \emptyset\right\rangle=\operatorname{det}_{1 \leq i, j \leq s}\left\langle\emptyset\left|\mathcal{T}\left(\Psi\left(\alpha_{i}\right), \Psi^{*}\left(\beta_{j}\right)\right)\right| \emptyset\right\rangle
$$

used in 59 .

Let $\Pi$ be the set of partitions of $\{1, \ldots, 2 s\}$ into unordered pairs. An element $\tau \in \Pi$ can be canonically written $\tau=\left\{\left\{i_{1}, j_{1}\right\}, \ldots,\left\{i_{s}, j_{s}\right\}\right\}$ with $i_{1}<i_{2}<\cdots<i_{s}$ and $i_{t}<j_{t}$ for all $t$, and viewed as a permutation

$$
\tau=\left(\begin{array}{ccccc}
1 & 2 & \cdots & 2 s-1 & 2 s \\
i_{1} & j_{1} & \cdots & i_{s} & j_{s}
\end{array}\right)
$$

which allows to define its sign $\epsilon(\tau)$. Recall that the Pfaffian of a $2 s \times 2 s$ antisymmetric matrix $\mathcal{A}=\left(\mathcal{A}_{i j}\right)_{1 \leq i, j \leq 2 s}$ is defined as

$$
\operatorname{Pf}(\mathcal{A})=\sum_{\tau \in \Pi} \epsilon(\tau) \prod_{t=1}^{s} \mathcal{A}_{\tau(2 t-1), \tau(2 t)} .
$$

Let $F$ be the space of (countably infinite) linear combinations of $\psi_{k}$ 's and $\psi_{k}^{*}$ 's.

Proposition 24 (Wick's formula). For $X_{1}, \ldots, X_{2 s}$ elements of $F$, we have

$$
\left\langle\emptyset\left|X_{1} \cdots X_{2 s}\right| \emptyset\right\rangle=\operatorname{Pf}(\mathcal{A})
$$

where $\mathcal{A}$ is the $2 s \times 2 s$ antisymmetric matrix such that $\mathcal{A}_{i j}=\left\langle\emptyset\left|X_{i} X_{j}\right| \emptyset\right\rangle$ for $i<j$.

Proof. Let $F^{+}$(resp. $F^{-}$) be the vector space spanned by the $\psi_{k}$ with $k>0$ and the $\psi_{k}^{*}$ with $k<0$ (resp. the $\psi_{k}$ with $k<0$ and the $\psi_{k}^{*}$ with $k>0$ ), so that $F=F^{+} \oplus F^{-}$. For $X \in F$, we denote by $X^{+}$and $X^{-}$its projections on these two subspaces: observe that $\langle\emptyset| X^{+}=0$ and $X^{-}|\emptyset\rangle=0$. 
Let $X_{1}, \ldots, X_{2 s}$ be elements of $F$. Observe that, by proposition $9\left\{X_{i}^{-}, X_{j}^{+}\right\}$is a scalar for any $i<j$, and

$$
\begin{aligned}
\left\langle\emptyset\left|X_{i} X_{j}\right| \emptyset\right\rangle & =\left\langle\emptyset\left|X_{i}^{+} X_{j}^{+}+X_{i}^{+} X_{j}^{-}+X_{i}^{-} X_{j}^{-}+X_{i}^{-} X_{j}^{+}\right| \emptyset\right\rangle \\
& =\left\langle\emptyset\left|X_{i}^{+} X_{j}^{+}+X_{i}^{+} X_{j}^{-}+X_{i}^{-} X_{j}^{-}+\left\{X_{i}^{-}, X_{j}^{+}\right\}-X_{j}^{+} X_{i}^{-}\right| \emptyset\right\rangle \\
& =\left\{X_{i}^{-}, X_{j}^{+}\right\} .
\end{aligned}
$$

We proceed similarly to compute the left-hand side of 117$)$ : we write each of the $X_{i}$ as $X_{i}=X_{i}^{+}+X_{i}^{-}$, expand the product and get a sum of $2^{2 s}$ terms. In each of these terms, we move all the $X_{i}^{-}$to the right. To get a nonzero contribution, we must pair up each $X_{i}^{-}$with one $X_{j}^{+}$such that $i<j$, and multiply all the anticommutators obtained in this fashion. So each nonzero contribution is equal, up to a sign, to

$$
\prod_{t=1}^{s}\left\{X_{\tau(2 t-1)}^{-}, X_{\tau(2 t)}^{+}\right\}
$$

where $\tau \in \Pi$ is some partition into pairs of $\{1, \ldots, 2 s\}$. Furthermore, the sign of this contribution corresponds to the number of swaps needed to bring each $X_{\tau(2 t-1)}^{-}$ exactly to the left of $X_{\tau(2 t)}^{+}$, i.e. it is the signature of $\tau$. This yields exactly the right-hand side of 116 with $\mathcal{A}_{i j}=\left\{X_{i}^{-}, X_{j}^{+}\right\}=\left\langle\emptyset\left|X_{i} X_{j}\right| \emptyset\right\rangle$.

To prove the wanted identity 114 , we set $Y_{2 t-1}=\Psi\left(\alpha_{t}\right)$ and $Y_{2 t}=\Psi^{*}\left(\beta_{t}\right)$, $t=1, \ldots, s$. By the definition of natural ordering, there is a permutation $\sigma$ such that

$$
\mathcal{T}\left(Y_{1}, \ldots, Y_{2 s}\right)=\epsilon(\sigma) Y_{\sigma(1)} \cdots Y_{\sigma(2 s)} .
$$

By Proposition 24 and since $Y_{\sigma(i)} Y_{\sigma(j)}=\mathcal{T}\left(Y_{\sigma(i)}, Y_{\sigma(j)}\right)$ for any $i<j$, we have

$$
\begin{aligned}
\left\langle\emptyset\left|\mathcal{T}\left(Y_{1}, \ldots, Y_{2 s}\right)\right| \emptyset\right\rangle & =\epsilon(\sigma) \operatorname{Pf}_{1 \leq i, j \leq 2 s}\left\langle\emptyset\left|\mathcal{T}\left(Y_{\sigma(i)}, Y_{\sigma(j)}\right)\right| \emptyset\right\rangle \\
& =\operatorname{Pf}_{1 \leq i, j \leq 2 s}\left\langle\emptyset\left|\mathcal{T}\left(Y_{i}, Y_{j}\right)\right| \emptyset\right\rangle
\end{aligned}
$$

(when we simultaneously permute the rows and columns of an antisymmetric matrix, the Pfaffian is multiplied by the sign of the permutation). Finally, we observe that $\left\langle\emptyset\left|\mathcal{T}\left(Y_{i}, Y_{j}\right)\right| \emptyset\right\rangle$ is nonzero if and only if $i, j$ have different parities, which allows to rewrite the Pfaffian as the wanted determinant without sign.

\section{REFERENCES}

[All14] Nicolas Allegra. Exact solution of the 2d dimer model: Corner free energy, correlation functions and combinatorics, 2014, arXiv:1410.4131 [cond-mat.stat-mech].

[AZ13] Alexander Alexandrov and Anton Zabrodin. Free fermions and tau-functions. Journal of Geometry and Physics, 67(0):37-80, 2013, arXiv:1212.6049 [math-ph].

$\left[\mathrm{BBB}^{+} 14\right]$ Dan Betea, Cédric Boutillier, Jérémie Bouttier, Guillaume Chapuy, Sylvie Corteel, and Mirjana Vuletić. Perfect sampling algorithm for Schur processes, 2014, arXiv:1407.3764 [math.PR].

[BC14] Alexei Borodin and Ivan Corwin. Macdonald processes. Probab. Theory Related Fields, 158(1-2):225-400, 2014, arXiv:1111.4408 [math.PR].

[BCC14] Jérémie Bouttier, Guillaume Chapuy, and Sylvie Corteel. From Aztec diamonds to pyramids: steep tilings, 2014, arXiv:1407.0665 [math.CO].

[BF14] Alexei Borodin and Patrik L. Ferrari. Anisotropic growth of random surfaces in $2+1$ dimensions. Comm. Math. Phys., 325(2):603-684, 2014, arXiv:0804.3035 [math-ph].

[BF15] Alexei Borodin and Patrik L. Ferrari. Random tilings and Markov chains for interlacing particles, 2015, arXiv:1506.03910 [math-ph].

[BK72] Edward A. Bender and Donald E. Knuth. Enumeration of plane partitions. J. Combinatorial Theory Ser. A, 13:40-54, 1972.

[BMRT12] Cédric Boutillier, Sevak Mkrtchyan, Nicolai Reshetikhin, and Peter Tingley. Random skew plane partitions with a piecewise periodic back wall. Ann. Henri Poincaré, 13(2):271-296, 2012, arXiv:0912.3968 [math-ph]. 
[Bor07] Alexei Borodin. Periodic Schur process and cylindric partitions. Duke Math. J., 140(3):391-468, 2007, arXiv:math/0601019 [math.CO].

[Bor11] Alexei Borodin. Schur dynamics of the Schur processes. Adv. Math., 228(4):2268-2291, 2011, arXiv:1001.3442 [math.CO].

[BP11] Yuliy Baryshnikov and Robin Pemantle. Asymptotics of multivariate sequences, part III: Quadratic points. Adv. Math., 228(6):3127-3206, 2011.

[BR05] Alexei Borodin and Eric M. Rains. Eynard-Mehta theorem, Schur process, and their Pfaffian analogs. J. Stat. Phys., 121(3-4):291-317, 2005, arXiv:math-ph/0409059.

[BS10] Alexei Borodin and Senya Shlosman. Gibbs ensembles of nonintersecting paths. Comm. Math. Phys., 293(1):145-170, 2010, arXiv:0804.0564 [math-ph].

[CEP96] Henry Cohn, Noam Elkies, and James Propp. Local statistics for random domino tilings of the Aztec diamond. Duke Math. J., 85(1):117-166, 1996, arXiv:math/0008243 [math.CO].

[CSV11] Sylvie Corteel, Cyrille Savelief, and Mirjana Vuletić. Plane overpartitions and cylindric partitions. J. Combin. Theory Ser. A, 118(4):1239-1269, 2011, arXiv:0903.2039 [math.CO].

[CY14] Sunil Chhita and Benjamin Young. Coupling functions for domino tilings of Aztec diamonds. Adv. Math., 259:173-251, 2014, arXiv:1302.0615 [math.CO].

[DFR12] Philippe Di Francesco and Nicolai Reshetikhin. Asymptotic shapes with free boundaries. Comm. Math. Phys., 309(1):87-121, 2012, arXiv:0908.1630 [math-ph].

[DFSG14] Philippe Di Francesco and Rodrigo Soto-Garrido. Arctic curves of the octahedron equation. J. Phys. A, 47(28):285204, 34, 2014, arXiv:1402.4493 [math-ph].

[Du11] Peng Du. The Aztec Diamond Edge-Probability Generating Function, 2011. Master Thesis, University of Pennsylvania.

[Dub11] Julien Dubédat. Exact bosonization of the Ising model, 2011, arXiv:1112.4399 [math.PR].

[EKLP92a] Noam Elkies, Greg Kuperberg, Michael Larsen, and James Propp. Alternatingsign matrices and domino tilings. I. J. Algebraic Combin., 1(2):111-132, 1992, arXiv:math/9201305 [math.CO].

[EKLP92b] Noam Elkies, Greg Kuperberg, Michael Larsen, and James Propp. Alternatingsign matrices and domino tilings. II. J. Algebraic Combin., 1(3):219-234, 1992, arXiv:math/9201305 [math.CO].

[FW09] Omar Foda and Michael Wheeler. Hall-Littlewood plane partitions and KP. Int. Math. Res. Not. IMRN, (14):2597-2619, 2009, arXiv:0809.2138 [math-ph].

[GMT14] Alessandro Giuliani, Vieri Mastropietro, and Fabio Lucio Toninelli. Height fluctuations in interacting dimers, 2014, arXiv:1406.7710 [math.PR].

[Hel00] H. Helfgott. Edge Effects on Local Statistics in Lattice Dimers: A Study of the Aztec Diamond (Finite Case). ArXiv Mathematics e-prints, July 2000, math/0007136.

[ID89] Claude Itzykson and Jean-Michel Drouffe. Statistical field theory. Vol. 1. Cambridge Monographs on Mathematical Physics. Cambridge University Press, Cambridge, 1989. From Brownian motion to renormalization and lattice gauge theory.

[Joh02] Kurt Johansson. Non-intersecting paths, random tilings and random matrices. Probab. Theory Related Fields, 123(2):225-280, 2002, arXiv:math/0011250 [math.PR].

[Joh05] Kurt Johansson. The arctic circle boundary and the Airy process. Ann. Probab., 33(1):1-30, 2005

[JPS98] William Jokusch, James Propp, and Peter Shor. Random Domino Tilings and the Arctic Circle Theorem, 1998, arXiv:math/9801068 [math.CO].

[Kac90] Victor G. Kac. Infinite-dimensional Lie algebras. Cambridge University Press, Cambridge, third edition, 1990.

[Kas61] P. W. Kasteleyn. The statistics of dimers on a lattice : I. The number of dimer arrangements on a quadratic lattice. Physica, 27:1209-1225, December 1961.

[Kas67] P. W. Kasteleyn. Graph theory and crystal physics. In Graph Theory and Theoretical Physics, pages 43-110. Academic Press, London, 1967.

[Ken97] Richard Kenyon. Local statistics of lattice dimers. Ann. Inst. H. Poincaré Probab. Statist., 33(5):591-618, 1997, arXiv:math/0105054 [math.CO].

[Ken05] Richard Kenyon. Talk given at the workshop on Random partitions and CalabiYau crystals, Amsterdam, 2005. Slides available at http://www.math.brown.edu/ $\sim$ rkenyon/talks/pyramids.pdf

$\left[\mathrm{KKM}^{+}{ }^{11}\right]$ N. Kitanine, K. K. Kozlowski, J.-M. Maillet, N. A. Slavnov, and V. Terras. A form factor approach to the asymptotic behavior of correlation functions in critical models. Journal of Statistical Mechanics: Theory and Experiment, 2011(12):P12010, 2011, arXiv:1110.0803 [hep-th]. 
[Mac04] Percy A. MacMahon. Combinatory analysis. Vol. I, II (bound in one volume). Dover Phoenix Editions. Dover Publications, Inc., Mineola, NY, 2004. Reprint of it An introduction to combinatory analysis (1920) and it Combinatory analysis. Vol. I, II (1915, 1916).

[MJD00] T. Miwa, M. Jimbo, and E. Date. Solitons, volume 135 of Cambridge Tracts in Mathematics. Cambridge University Press, Cambridge, 2000. Differential equations, symmetries and infinite-dimensional algebras, Translated from the 1993 Japanese original by Miles Reid.

[Oka10] Soichi Okada. $(q, t)$-deformations of multivariate hook product formulae. J. Algebraic Combin., 32(3):399-416, 2010, arXiv:0909.0086 [math.CO].

[Oko01] Andrei Okounkov. Infinite wedge and random partitions. Selecta Math. (N.S.), 7(1):57-81, 2001, arXiv:math/9907127 [math.RT].

[Oko02] Andrei Okounkov. Symmetric functions and random partitions. In Symmetric functions 2001: surveys of developments and perspectives, volume 74 of NATO Sci. Ser. II Math. Phys. Chem., pages 223-252. Kluwer Acad. Publ., Dordrecht, 2002, arXiv:math/0309074 [math.CO].

[OR03] Andrei Okounkov and Nikolai Reshetikhin. Correlation function of Schur process with application to local geometry of a random 3-dimensional Young diagram. J. Amer. Math. Soc., 16(3):581-603 (electronic), 2003, arXiv:math/0107056 [math.CO].

[OR07] Andrei Okounkov and Nicolai Reshetikhin. Random skew plane partitions and the Pearcey process. Comm. Math. Phys., 269(3):571-609, 2007, arXiv:math/0503508 [math.CO].

[Pan14] Greta Panova. Lozenge tilings with free boundaries, 2014, arXiv:1408.0417 [math.PR].

[Per69] J. K. Percus. One more technique for the dimer problem. J. Math. Phys., 10:1881, 1969.

[Pet14] Leonid Petrov. Asymptotics of random lozenge tilings via Gelfand-Tsetlin schemes. Probab. Theory Related Fields, 160(3-4):429-487, 2014, arXiv:1202.3901 [math.PR].

[Pro97] James Propp. Talk given at the American Mathematical Society Meeting in San Diego, January 1997. Slides available at http://jamespropp.org/san_diego.pdf.

[Pro02] James Propp. Lattice structure for orientations of graphs, 2002, arXiv:math/0209005 [math.CO].

[Sta99] Richard P. Stanley. Enumerative combinatorics. Vol. 2, volume 62 of Cambridge Studies in Advanced Mathematics. Cambridge University Press, Cambridge, 1999. With a foreword by Gian-Carlo Rota and appendix 1 by Sergey Fomin.

[Tin11] Peter Tingley. Notes on Fock space, 2011. http://webpages.math.luc.edu/ ptingley/lecturenotes/Fock_space-2010.pdf

[Vul07] Mirjana Vuletić. The shifted Schur process and asymptotics of large random strict plane partitions. Int. Math. Res. Not. IMRN, (14):Art. ID rnm043, 53, 2007, arXiv:math-ph/0702068.

[Vul09] Mirjana Vuletić. A generalization of MacMahon's formula. Trans. Amer. Math. Soc., 361(5):2789-2804, 2009, arXiv:0707.0532 [math.CO].

[Vul13] Mirjana Vuletić. The Gaussian free field and strict plane partitions. In 25th International Conference on Formal Power Series and Algebraic Combinatorics (FPSAC 2013), Discrete Math. Theor. Comput. Sci. Proc., AS, pages 1041-1052. Assoc. Discrete Math. Theor. Comput. Sci., Nancy, 2013.

[Yan91] Bo-Yin Yang. Two enumeration problems about the Aztec diamonds. ProQuest LLC, Ann Arbor, MI, 1991. Thesis (Ph.D.)-Massachusetts Institute of Technology, available online at http://hdl.handle.net/1721.1/13937.

[You09] Ben Young. Computing a pyramid partition generating function with dimer shuffling. J. Combin. Theory Ser. A, 116(2):334-350, 2009, arXiv:0709.3079 [math.CO].

[You10] Benjamin Young. Generating functions for colored 3D Young diagrams and the Donaldson-Thomas invariants of orbifolds. Duke Math. J., 152(1):115-153, 2010, arXiv:0802.3948 [math.CO]. With an appendix by Jim Bryan. 
Laboratoire de Probabilités et Modèles Aléatoires, UMR 7599, Université Pierre et Marie Curie, 4 Place Jussieu, F-75005 Paris

E-mail address: cedric.boutillier@upmc.fr

Institut de Physique Théorique, Université Paris-Saclay, CEA, CNRS, F-91191 GifSur-Yvette and Département de Mathématiques et Applications, École normale supérieure, 45 Rue d'Ulm, F-75231 Paris Cedex 05

E-mail address: jeremie.bouttier@cea.fr

LiAfA, CNRS et Université Paris Diderot, Case 7014, F-75205 Paris Cedex 13

E-mail address: guillaume.chapuy@liafa.univ-paris-diderot.fr

LiAfA, CNRS et Université Paris Diderot, Case 7014, F-75205 Paris Cedex 13

E-mail address: corteel@liafa.univ-paris-diderot.fr

Mathematics Departement, Brown University, Box 1917, 151 Thayer Street, ProviDENCE, RI 02912

E-mail address: sanjay_ramassamy@brown.edu 